Supporting Information

\title{
Role of Amino-Acid Residues for Dioxygen Activation in the Second Coordination Sphere of the Dicopper Site of pMMO
}

Mayuko Miyanishi, Tsukasa Abe, Yuta Hori, Yoshihito Shiota, and Kazunari Yoshizawa*

Institute for Materials Chemistry and Engineering and IRCCS, Kyushu University, Fukuoka 819-0395,

Japan

* To whom all correspondence should be addressed (e-mail: kazunari@ms.ifoc.kyushu-u.ac.jp). 


\section{Table of Contents}

Figure S1. Energy profile for the PT from $\mathrm{Tyr}_{374}$ to the $\mathrm{Cu}_{2} \mathrm{O}_{2}$ core in $\mathbf{P O}^{\mathbf{T}}$ in the open-shell singlet state.

Figure S2. Energy profile for the $\mathrm{O}-\mathrm{O}$ bond cleavage from $\mathbf{P O}^{\mathbf{T}}$ in the singlet state.

Figure S3. Energy profile for the $\mathrm{O}-\mathrm{O}$ bond cleavage from Int1a in the open-shell singlet state.

Figure S4. Plots of spin density against the $\mathrm{Cu}-\mathrm{O}_{\mathrm{tyr}}$ bond distance for the $\mathrm{Cu}(1)$ (green), $\mathrm{Cu}(2)(\mathrm{red})$ and $\mathrm{O}_{\text {tyr }}$ (blue) in the open-shell singlet state.

Figure S5. Plots of $\triangle E S P$ charges against the $\mathrm{Cu}-\mathrm{O}_{\text {tyr }}$ bond distance for the $\mathrm{Cu}_{2} \mathrm{O}_{2}$ core (red) and Tyr374 residue (blue) in the open-shell singlet state.

Figure S6. Optimized $\mathrm{Cu}_{2} \mathrm{O}_{2}$ core structures with Glu35 and Tyr374 of intermediates $\mathbf{P O}{ }^{\mathrm{G}}, \mathbf{P O}^{\mathrm{T}}$, Intıa, Intıb, Int2, Int3, BO, and $\mathbf{O H O}$ and transition states $\mathbf{T S} \mathbf{1}, \mathbf{T S} \mathbf{2}$, and $\mathbf{T S}_{\mathbf{3}}$ in the triplet state.

Table S1. Calculated Mulliken spin populations in the triplet state.

Table S2. Calculated electrostatic potential fit (ESP) charge in the triplet state.

Table S3. Cartesian coordinates of $\mathbf{P O} \mathbf{G}^{\mathbf{G}}$ in the triplet state.

Table S4. Cartesian coordinates of $\mathbf{P} \mathbf{O}^{\mathbf{G}}$ in the open-shell singlet state.

Table S5. Cartesian coordinates of $\mathbf{P O}^{\mathbf{T}}$ in the triplet state.

Table S6. Cartesian coordinates of $\mathbf{P O}^{\mathbf{T}}$ in the open-shell singlet state.

Table S7. Cartesian coordinates of Int1a in the triplet state. 
Table S8. Cartesian coordinates of Int1a in the open-shell singlet state.

Table S9. Cartesian coordinates of Int1b in the triplet state.

Table S10. Cartesian coordinates of Int1b in the open-shell singlet state.

Table S11. Cartesian coordinates of TS1 in the triplet state.

Table S12. Cartesian coordinates of TS1 in the open-shell singlet state.

Table S13. Cartesian coordinates of Int2 in the triplet state.

Table S14. Cartesian coordinates of Int2 in the open-shell singlet state.

Table S15. Cartesian coordinates of TS2 in the triplet state.

Table S16. Cartesian coordinates of TS2 in the open-shell singlet state.

Table S17. Cartesian coordinates of Int3 in the triplet state.

Table S18. Cartesian coordinates of Int3 in the open-shell singlet state.

Table S19. Cartesian coordinates of TS3 in the triplet state.

Table S20. Cartesian coordinates of TS3 in the open-shell singlet state.

Table S21. Cartesian coordinates of BO in the triplet state.

Table S22. Cartesian coordinates of BO in the open-shell singlet state.

Table S23. Cartesian coordinates of OHO in the triplet state.

Table S24. Cartesian coordinates of OHO in the open-shell singlet state. 

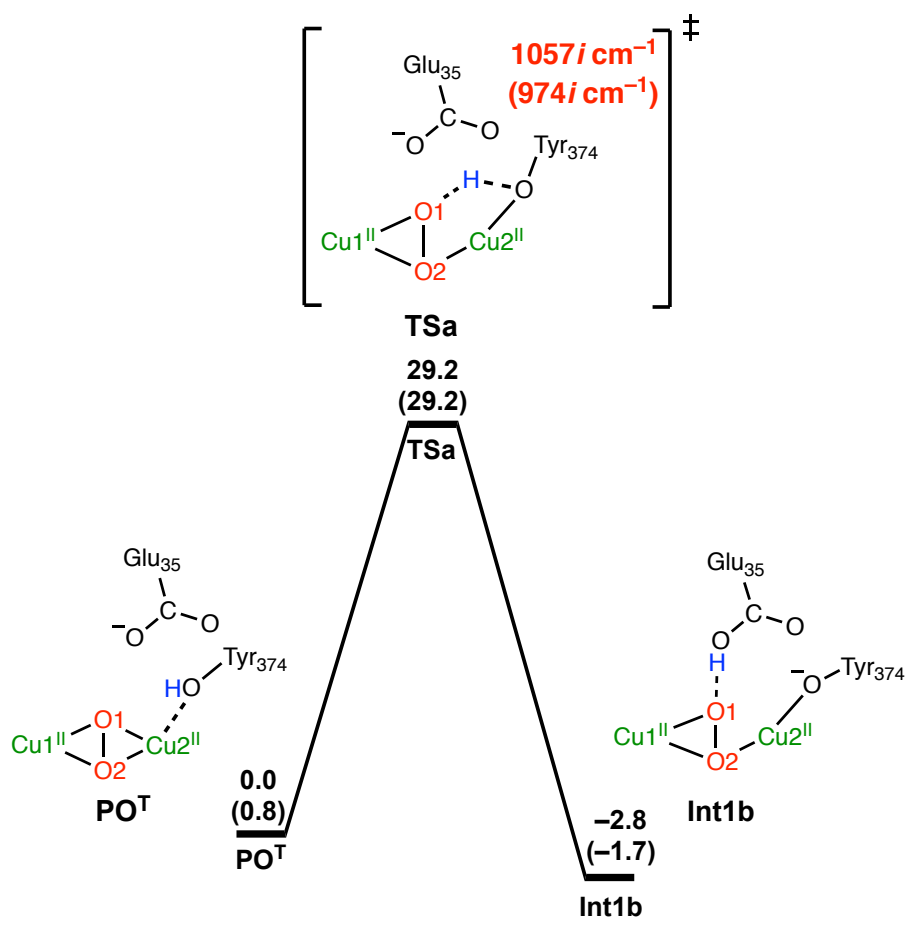

Figure S1. Energy profile for the PT from $\mathrm{Tyr}_{374}$ to the $\mathrm{Cu}_{2} \mathrm{O}_{2}$ core in $\mathbf{P O}^{\mathbf{T}}$ in the open-shell singlet state. The relative energies with respect to $\mathbf{P O}^{\mathbf{T}}$ are in $\mathrm{kcal} / \mathrm{mol}$. Those of the triplet state are shown in the parentheses.
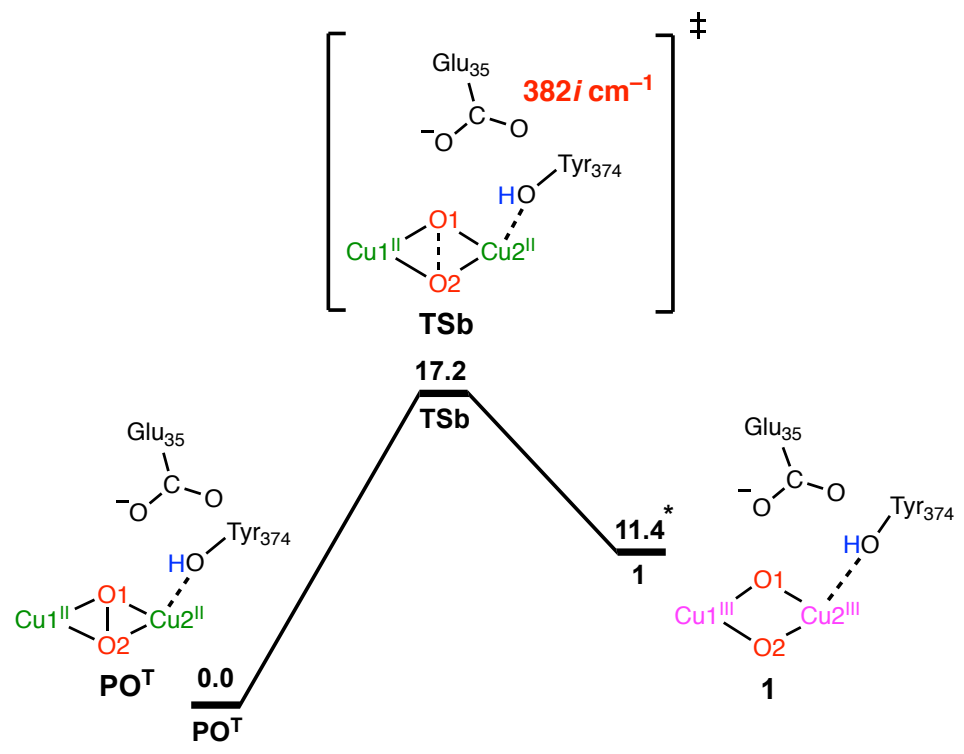

Figure S2. Energy profile for the $\mathrm{O}-\mathrm{O}$ bond cleavage from $\mathbf{P O}^{\mathrm{T}}$ in the open-shell singlet state. The relative energies with respect to $\mathbf{P O}^{\mathbf{T}}$ are in $\mathrm{kcal} / \mathrm{mol}$. Asterisk denotes that the energy of $\mathbf{1}$ is calculated in the closed-shell singlet state. 

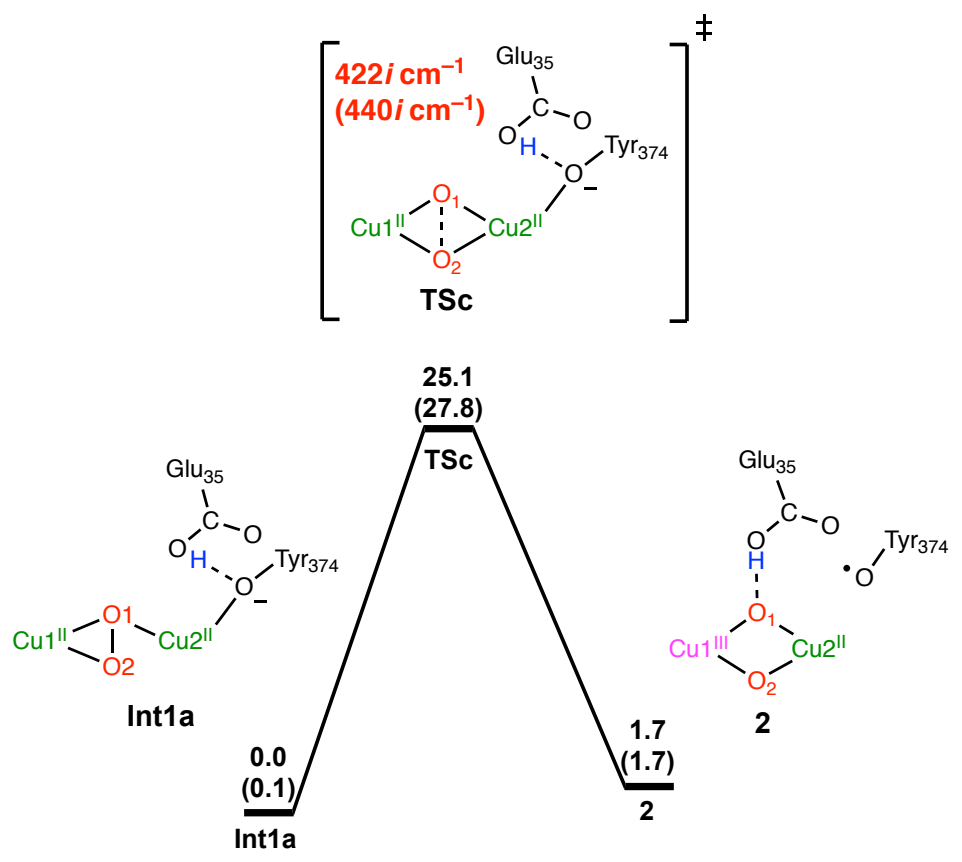

Figure S3. Energy profile for the $\mathrm{O}-\mathrm{O}$ bond cleavage from Int1a in the open-shell singlet state. The relative energies with respect to Int1a are in $\mathrm{kcal} / \mathrm{mol}$. Those of the triplet state are shown in the parentheses.

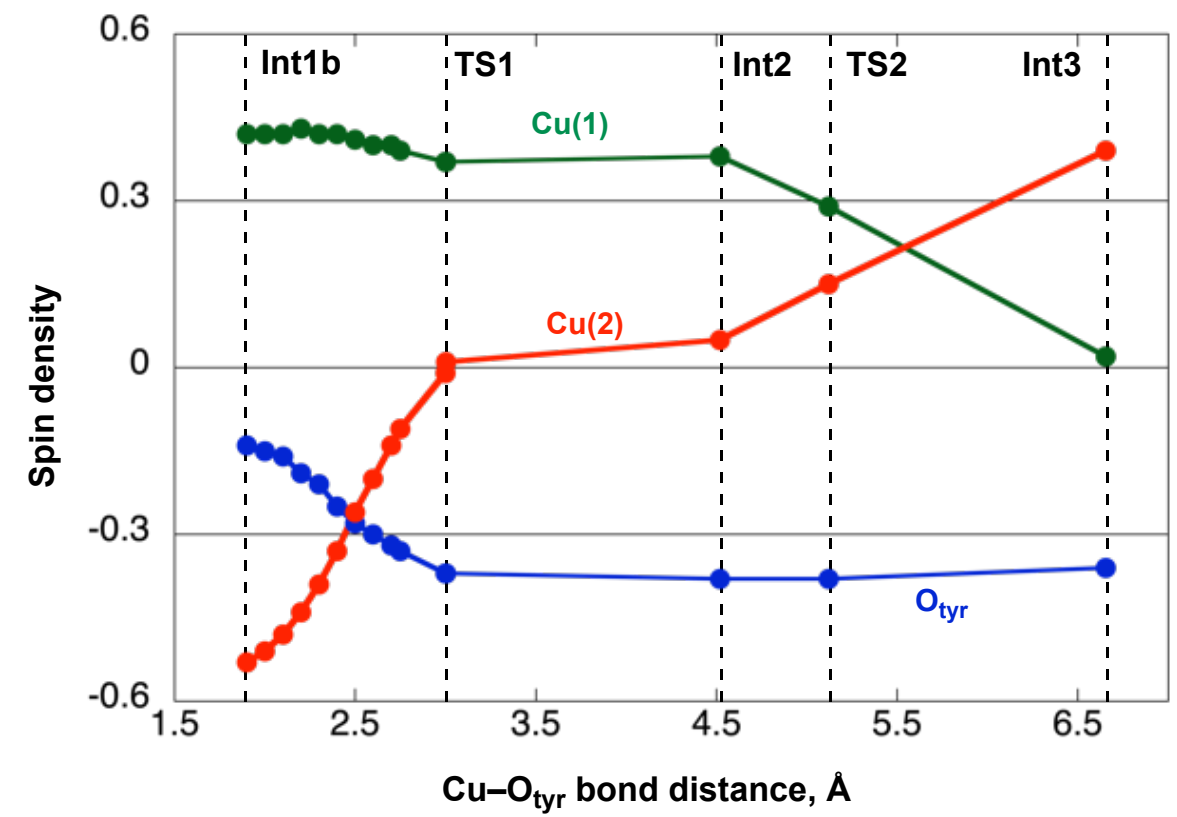

Figure S4. Plots of the spin densities against the $\mathrm{Cu}-\mathrm{O}_{\mathrm{tyr}}$ bond distance for the $\mathrm{Cu}(1)$ (green), $\mathrm{Cu}(2)$ (red) and $\mathrm{O}_{\mathrm{tyr}}$ (blue) in the open-shell singlet state. 


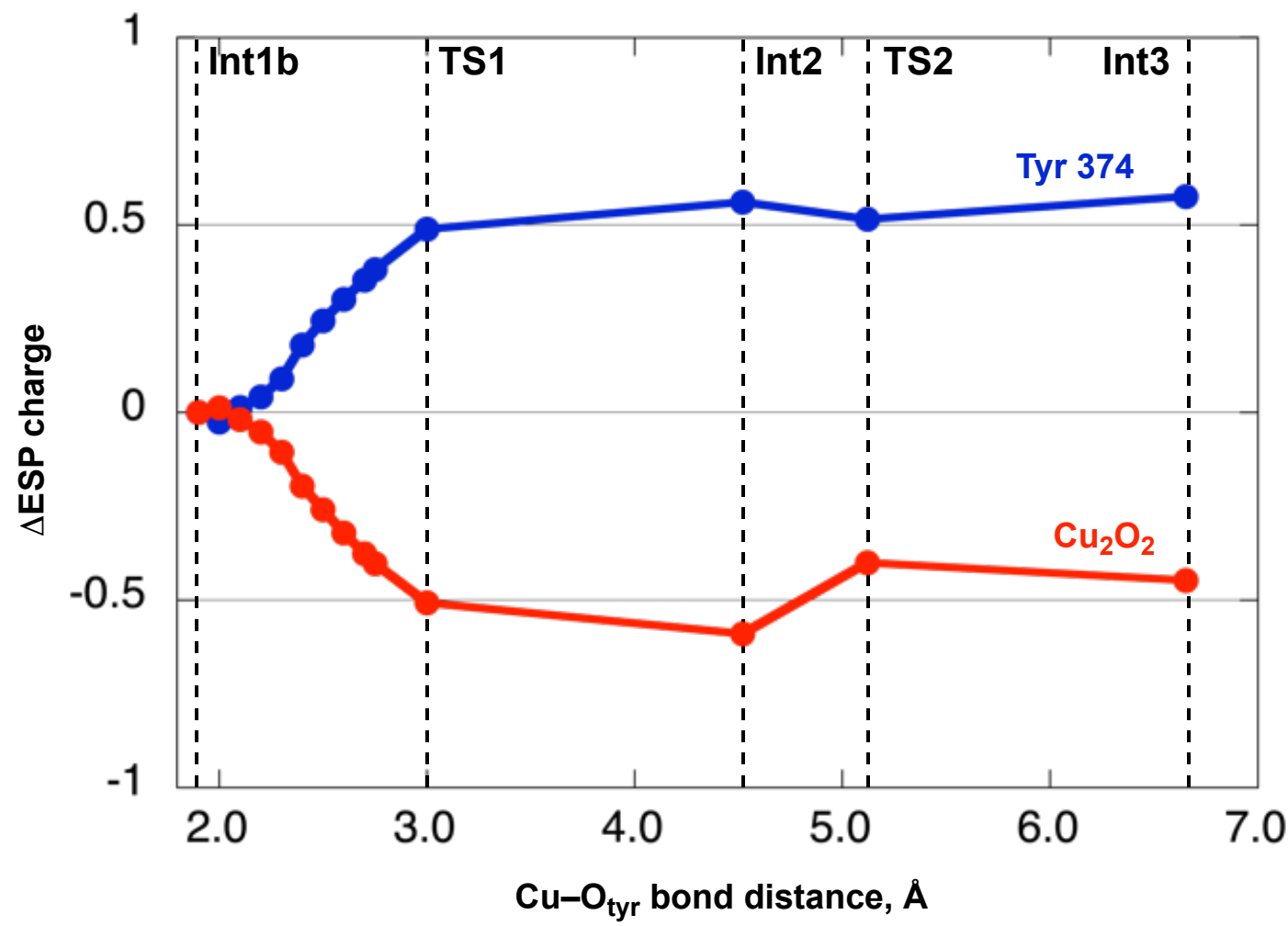

Figure S5. Plots of $\triangle E S P$ charges against the $\mathrm{Cu}-\mathrm{O}_{\text {tyr }}$ bond distance for the $\mathrm{Cu}_{2} \mathrm{O}_{2}$ core (red) and Tyr374 residue (blue) in the open-shell singlet state. $\Delta \mathrm{ESP}=R_{\mathrm{x}}-R_{\mathrm{IntP}}\left(R_{\mathrm{x}}\right.$ : ESP charges at each $\mathrm{Cu}-\mathrm{O}_{\mathrm{tyr}}$ bond distance, $R_{\mathrm{IntP}} *$ : ESP charges at Int1b). 

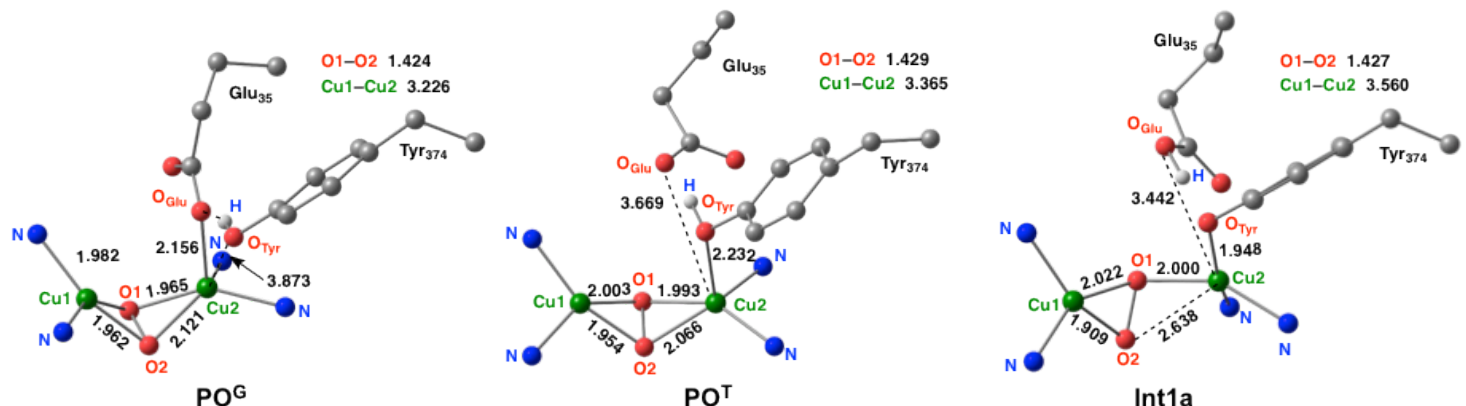

Int1a

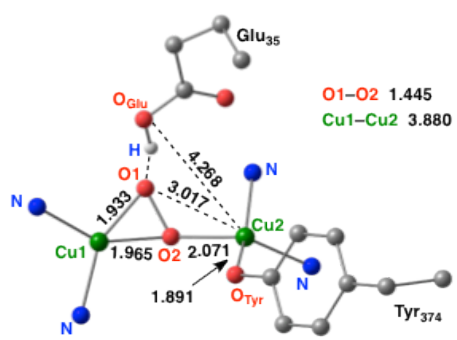

Int1b

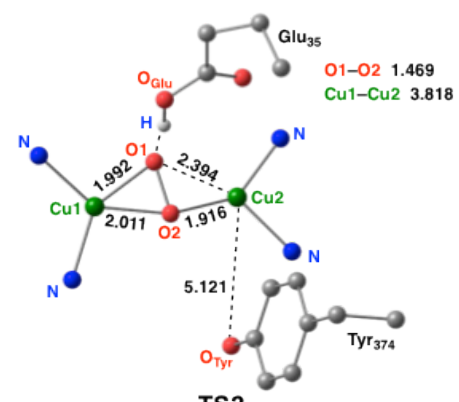

TS2

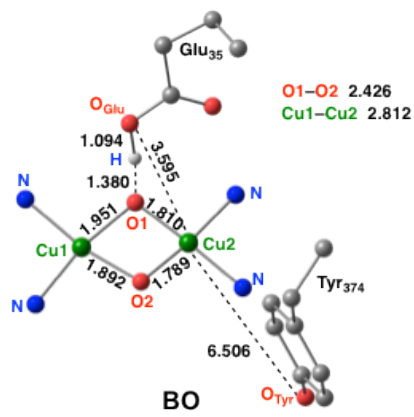

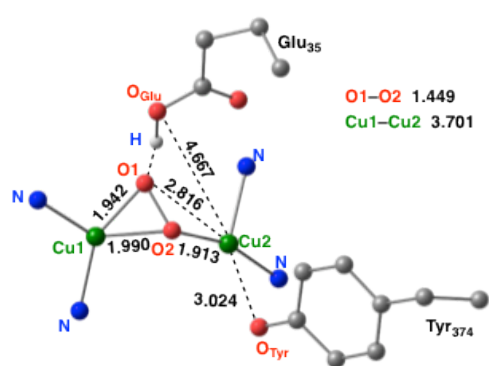

TS1

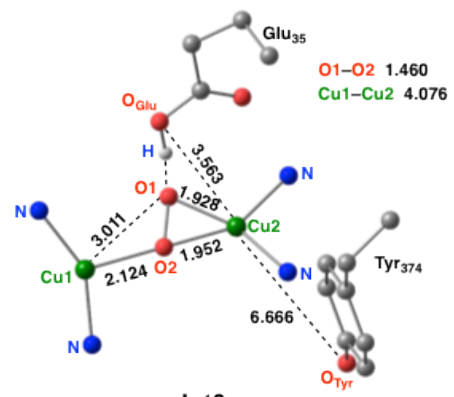

Int3

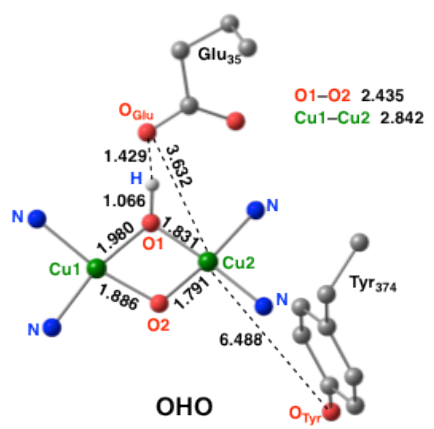

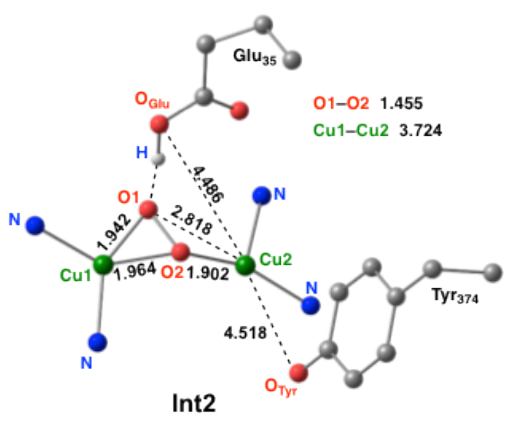

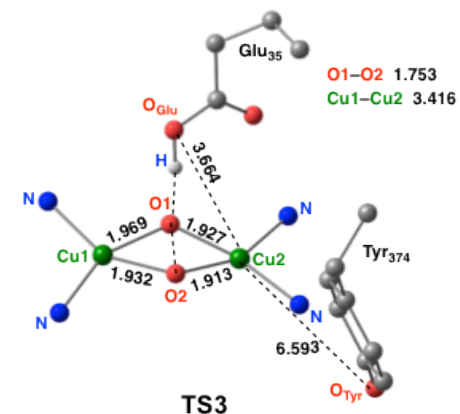

TS3

Figure S6. Optimized $\mathrm{Cu}_{2} \mathrm{O}_{2}$ core structures with Glu35 and Tyr374 of intermediates $\mathbf{P O}^{\mathbf{G}}, \mathbf{P O}^{\mathbf{T}}$, Int1a, Int1b, Int2, Int3, BO, and OHO and transition states TS1, TS2, and TS3 in the triplet state. The units are in angstroms. His33, His137, His139, and hydrogen atoms are omitted for clarity. 
Table S1. Calculated Mulliken spin populations in the triplet state.

\begin{tabular}{cccccc}
\hline Species & Cu1 & Cu2 & O1 & O2 & O $_{\text {Tyr }}$ \\
\hline PO $^{\mathbf{G}}$ & 0.42 & 0.50 & 0.44 & 0.41 & 0.00 \\
PO $^{\text {T }}$ & 0.42 & 0.51 & 0.43 & 0.40 & 0.00 \\
Int1a & 0.37 & 0.53 & 0.41 & 0.36 & 0.09 \\
Int1b & 0.41 & 0.54 & 0.27 & 0.30 & 0.15 \\
TS1 & 0.37 & 0.11 & 0.24 & 0.26 & 0.37 \\
Int2 & 0.38 & 0.05 & 0.26 & 0.23 & 0.38 \\
TS2 & 0.29 & 0.15 & 0.28 & 0.22 & 0.38 \\
Int3 & 0.03 & 0.39 & 0.25 & 0.24 & 0.36 \\
TS3 & 0.31 & 0.39 & 0.10 & 0.06 & 0.36 \\
BO & 0.54 & -0.12 & 0.20 & 0.31 & 0.36 \\
OHO & 0.57 & -0.11 & 0.14 & 0.33 & 0.36 \\
\hline
\end{tabular}

Table S2. Calculated electrostatic potential fit (ESP) charge in the triplet state.

\begin{tabular}{cccc}
\hline Species & $\mathbf{C u}_{2} \mathbf{O}_{2}{ }^{a}$ & Glu35 & Tyr374 \\
\hline PO & $1.79 \mathrm{e}$ & $-0.70 \mathrm{e}$ & $-0.09 \mathrm{e}$ \\
Int1a & $1.59 \mathrm{e}$ & $0.11 \mathrm{e}$ & $-0.70 \mathrm{e}$ \\
Int1b & $1.42 \mathrm{e}$ & $0.04 \mathrm{e}$ & $-0.46 \mathrm{e}$ \\
TS1 & $0.88 \mathrm{e}$ & $0.14 \mathrm{e}$ & $-0.02 \mathrm{e}$ \\
Int2 & $0.81 \mathrm{e}$ & $0.15 \mathrm{e}$ & $0.04 \mathrm{e}$ \\
TS2 & $0.99 \mathrm{e}$ & $0.01 \mathrm{e}$ & $-0.00 \mathrm{e}$ \\
Int3 & $0.99 \mathrm{e}$ & $-0.05 \mathrm{e}$ & $0.06 \mathrm{e}$ \\
TS3 & $0.95 \mathrm{e}$ & $0.00 \mathrm{e}$ & $0.05 \mathrm{e}$ \\
BO & $0.98 \mathrm{e}$ & $-0.05 \mathrm{e}$ & $0.07 \mathrm{e}$ \\
OHO & $1.57 \mathrm{e}^{b}$ & $-0.64 \mathrm{e}$ & $0.07 \mathrm{e}$ \\
\hline
\end{tabular}

${ }^{a}$ The $\mathrm{Cu}_{2} \mathrm{O}_{2}$ core including His33, His137, and His $139 .{ }^{b}$ The protonated $\mathrm{Cu}_{2} \mathrm{O}_{2}$ core. 
Table S3. Cartesian coordinates of $\mathrm{PO}^{\mathrm{G}}$ in the triplet state.
Atom $\mathrm{x} \quad \mathrm{y} \quad \mathrm{z}$
$\begin{array}{llll}\mathrm{N} & 0.693774 & 3.821165 & -0.182279\end{array}$
$\begin{array}{llll}\text { C } & 2.047542 & 4.404454 & -0.339424\end{array}$
H $\quad 2.040573 \quad 5.471381-0.080675$
C $2.582869 \quad 4.235064-1.774990$
C $2.940464 \quad 2.815374-2.118106$
$\begin{array}{llll}\mathrm{N} & 2.087401 & 1.747350 & -1.867785\end{array}$
$\begin{array}{llll}\text { C } & 4.093497 & 2.323284 & -2.688943\end{array}$
$\begin{array}{llll}\text { C } & 2.704844 & 0.641779 & -2.269221\end{array}$
N $\quad 3.919688 \quad 0.951986-2.778612$
$\begin{array}{llll}\mathrm{H} & 3.052808 & 2.671238 & 3.029858\end{array}$
C $3.212271 \quad 1.971184 \quad 3.859626$
H $\quad 4.002813 \quad 1.268466 \quad 3.567238$
C $\quad 1.910468 \quad 1.229706 \quad 4.215070$
C $\quad 1.335963 \quad 0.375552 \quad 3.069414$
C $\quad 0.715876 \quad 1.175702 \quad 1.917210$
$\begin{array}{llll}\text { O } & 0.738839 & 0.629179 & 0.739035\end{array}$
$\begin{array}{llll}\text { O } & 0.182495 & 2.287017 & 2.152825\end{array}$
$\begin{array}{llll}\mathrm{H} & -7.267418 & -0.531612 & -3.200791\end{array}$
$\begin{array}{llll}\text { C } & -6.516275 & -1.322448 & -3.113753\end{array}$
H $\quad-6.969542 \quad-2.159151 \quad-2.569824$
$\begin{array}{llll}\text { C } & -5.269751 & -0.783382 & -2.383625\end{array}$
$\begin{array}{llll}\text { C } & -4.160103 & -1.788118 & -2.202616\end{array}$
$\begin{array}{llll}\mathrm{N} & -2.974330 & -1.484809 & -1.530523\end{array}$
$\begin{array}{llll}\text { C } & -4.075241 & -3.089701 & -2.648302\end{array}$
$\begin{array}{llll}\text { C } & -2.201684 & -2.567074 & -1.578546\end{array}$
$\begin{array}{llll}\mathrm{N} & -2.835416 & -3.559637 & -2.245003\end{array}$
$\mathrm{H} \quad-6.916003 \quad-1.559934 \quad 2.552742$
$\begin{array}{llll}\text { C } & -6.310983 & -1.892671 & 3.403436\end{array}$
H $\quad-6.993772 \quad-2.237486 \quad 4.185777$
$\begin{array}{llll}\text { C } & -5.429252 & -0.753534 & 3.939428\end{array}$ 

$\begin{array}{llll}\text { C } & -4.455702 & -0.225130 & 2.923621\end{array}$
$\begin{array}{llll}\mathrm{N} & -3.550812 & 0.792664 & 3.205749\end{array}$
$\begin{array}{llll}\text { C } & -4.209433 & -0.547505 & 1.607447\end{array}$
$\begin{array}{llll}\text { C } & -2.810078 & 1.054677 & 2.100337\end{array}$
$\begin{array}{llll}\mathrm{N} & -3.190325 & 0.252917 & 1.109459\end{array}$
H $7.734293 \quad-3.799038-0.770252$
$\begin{array}{llll}\text { C } & 7.852369 & -3.540898 & 0.288589\end{array}$
$\begin{array}{llll}\mathrm{H} & 8.761747 & -4.027992 & 0.658782\end{array}$
$\begin{array}{llll}\text { C } & 6.623699 & -3.988646 & 1.110687\end{array}$
$\begin{array}{llll}\text { C } & 5.307169 & -3.354779 & 0.690637\end{array}$
$\begin{array}{llll}\text { C } & 5.014432 & -2.009708 & 0.982528\end{array}$
$\begin{array}{llll}\text { C } & 4.314838 & -4.097883 & 0.025064\end{array}$
$\begin{array}{llll}\text { C } & 3.782646 & -1.431105 & 0.644545\end{array}$
$\begin{array}{llll}\text { C } & 3.074039 & -3.539659 & -0.313551\end{array}$
$\begin{array}{llll}\text { C } & 2.789416 & -2.203010 & 0.010692\end{array}$
$\begin{array}{llll}\text { O } & 1.556524 & -1.695936 & -0.320138\end{array}$
$\mathrm{Cu} \quad 0.347547 \quad 1.931681 \quad-0.933366$
$\begin{array}{lllll}\mathrm{Cu} & -2.389737 & 0.241795 & -0.695930\end{array}$
$\mathrm{H} \quad 2.099418 \quad 0.571320 \quad 5.073070$
$\mathrm{H} \quad 1.150887 \quad 1.955757 \quad 4.525584$
$\begin{array}{llll}\mathrm{H} & 2.090281 & -0.311074 & 2.671201\end{array}$
$\begin{array}{llll}\mathrm{H} & 0.525689 & -0.259409 & 3.459083\end{array}$
$\mathrm{H} \quad 3.578376 \quad 2.549280 \quad 4.715369$
$\begin{array}{llll}\mathrm{H} & 6.809914 & -3.760225 & 2.168527\end{array}$
$\begin{array}{llll}\mathrm{H} & 6.531585 & -5.079477 & 1.044467\end{array}$
H $\quad 4.503905 \quad-5.142906 \quad-0.217565$
$\begin{array}{llll}\mathrm{H} & 5.755441 & -1.399508 & 1.498099\end{array}$
$\begin{array}{llll}\mathrm{H} & 2.310414 & -4.137810 & -0.806251\end{array}$
$\begin{array}{llll}\mathrm{H} & 3.583962 & -0.387694 & 0.882440\end{array}$
$\begin{array}{llll}\mathrm{H} & 1.363231 & -0.859105 & 0.183652\end{array}$
$\begin{array}{llll}\mathrm{H} & 8.000542 & -2.456493 & 0.354696\end{array}$
$\begin{array}{llll}\mathrm{H} & 0.477855 & 3.614053 & 0.805686\end{array}$
$\begin{array}{llll}\mathrm{H} & 2.714103 & 3.898518 & 0.366791\end{array}$ 

H $\quad 3.475209 \quad 4.858461 \quad-1.896815$
$\mathrm{H} \quad 1.834144 \quad 4.618156 \quad-2.483496$
H $\quad 4.991112 \quad 2.818984 \quad-3.027467$
H $2.320445 \quad-0.365096-2.170236$
$\mathrm{H} \quad 4.594074 \quad 0.282264 \quad-3.126308$
H $\quad-6.269554-1.661046-4.126340$
H $\quad-5.561598 \quad-0.398209-1.396510$
$\mathrm{H} \quad-4.866707 \quad 0.076295 \quad-2.936865$
H $\quad-4.769506 \quad-3.698264 \quad-3.207052$
H $\quad-1.205700 \quad-2.654944-1.160610$
H $\quad-2.459694 \quad-4.482823 \quad-2.420178$
$\begin{array}{llll}\mathrm{H} & -5.704802 & -2.747977 & 3.084985\end{array}$
$\begin{array}{llll}\mathrm{H} & -6.067660 & 0.070900 & 4.286491\end{array}$
H $\quad-4.872369-1.105111 \quad 4.819220$
$\begin{array}{llll}\mathrm{H} & -3.447362 & 1.264857 & 4.095607\end{array}$
$\begin{array}{llll}\mathrm{H} & -4.689115 & -1.300631 & 1.000261\end{array}$
$\begin{array}{llll}\mathrm{H} & -2.002737 & 1.779122 & 2.062000\end{array}$
H $\quad-0.016498 \quad 4.460291-0.539516$
$\begin{array}{llll}\text { O } & -1.612972 & 2.038542 & -1.007032\end{array}$
$\begin{array}{llll}\text { O } & -1.268954 & 1.123982 & -2.042921\end{array}$ 
Table S4. Cartesian coordinates of $\mathrm{PO}^{\mathrm{G}}$ in the open-shell singlet state.
Atom $\mathrm{X}$
$\mathrm{y}$
Z
N $\quad 0.721350 \quad 3.803165 \quad-0.305193$
C $2.077479 \quad 4.383754-0.454292$
H $\quad 2.064253 \quad 5.455269-0.216076$
C $\quad 2.636508 \quad 4.188061 \quad-1.877640$
$\begin{array}{llll}\text { C } & 2.993910 & 2.760822 & -2.187570\end{array}$
N $2.129063 \quad 1.703880-1.932966$
C $\quad 4.155039 \quad 2.249079 \quad-2.723495$
$\begin{array}{llll}\text { C } & 2.746595 & 0.585382 & -2.296493\end{array}$
$\begin{array}{llll}\mathrm{N} & 3.973716 & 0.877111 & -2.786727\end{array}$
$\mathrm{H} \quad 3.060450 \quad 2.753720 \quad 2.900249$
$\begin{array}{llll}\text { C } & 3.223024 & 2.092699 & 3.760856\end{array}$
H $3.995177 \quad 1.361656 \quad 3.489424$
C $\quad 1.915295 \quad 1.3951394 .176062$
$\begin{array}{llll}\text { C } & 1.308092 & 0.496836 & 3.082272\end{array}$
$\begin{array}{llll}\text { C } & 0.731440 & 1.259222 & 1.883269\end{array}$
$\begin{array}{llll}\text { O } & 0.805562 & 0.686619 & 0.723287\end{array}$
$\begin{array}{llll}\mathrm{O} & 0.185415 & 2.375747 & 2.071394\end{array}$
H $\quad-7.296598 \quad-0.635490 \quad-3.150665$
$\begin{array}{llll}\text { C } & -6.549157 & -1.426781 & -3.039078\end{array}$
H $\quad-7.001543 \quad-2.237437 \quad-2.456381$
$\begin{array}{llll}\text { C } & -5.292084 & -0.867679 & -2.346005\end{array}$
$\begin{array}{llll}\text { C } & -4.189136 & -1.873500 & -2.136793\end{array}$
$\begin{array}{llll}\mathrm{N} & -2.995517 & -1.549811 & -1.490307\end{array}$
$\begin{array}{llll}\text { C } & -4.114149 & -3.191777 & -2.532078\end{array}$
$\begin{array}{llll}\text { C } & -2.226405 & -2.635451 & -1.504187\end{array}$
$\begin{array}{llll}\mathrm{N} & -2.871503 & -3.650440 & -2.123921\end{array}$
$\begin{array}{llll}\mathrm{H} & -6.947418 & -1.417362 & 2.633352\end{array}$
$\begin{array}{llll}\text { C } & -6.335691 & -1.700929 & 3.497015\end{array}$
$\begin{array}{llll}\mathrm{H} & -7.012801 & -1.998868 & 4.303185\end{array}$
$\begin{array}{llll}\text { C } & -5.449080 & -0.533845 & 3.959485\end{array}$ 

$\begin{array}{llll}\text { C } & -4.483118 & -0.066129 & 2.907317\end{array}$
$\begin{array}{llll}\mathrm{N} & -3.559752 & 0.949793 & 3.130170\end{array}$
$\begin{array}{llll}\text { C } & -4.260519 & -0.451860 & 1.603935\end{array}$
$\begin{array}{llll}\text { C } & -2.830888 & 1.150064 & 2.004476\end{array}$
$\begin{array}{llll}\mathrm{N} & -3.237616 & 0.309742 & 1.056408\end{array}$
$\begin{array}{llll}\mathrm{H} & 7.689977 & -3.892620 & -0.617584\end{array}$
$\begin{array}{llll}\text { C } & 7.805814 & -3.618676 & 0.437546\end{array}$
$\begin{array}{llll}\mathrm{H} & 8.705717 & -4.113026 & 0.821187\end{array}$
$\begin{array}{llll}\text { C } & 6.565408 & -4.033568 & 1.258877\end{array}$
$\begin{array}{llll}\text { C } & 5.263914 & -3.385695 & 0.815461\end{array}$
$\begin{array}{llll}\text { C } & 4.984624 & -2.035483 & 1.096417\end{array}$
$\begin{array}{llll}\text { C } & 4.274796 & -4.118049 & 0.133610\end{array}$
$\begin{array}{llll}\text { C } & 3.768309 & -1.440884 & 0.731809\end{array}$
$\begin{array}{llll}\text { C } & 3.049338 & -3.543643 & -0.232422\end{array}$
$\begin{array}{llll}\text { C } & 2.777674 & -2.201393 & 0.080248\end{array}$
$\begin{array}{llll}\text { O } & 1.559922 & -1.678369 & -0.277116\end{array}$
$\begin{array}{llll}\mathrm{Cu} & 0.384804 & 1.913862 & -1.024829\end{array}$
$\begin{array}{lllll}\mathrm{Cu} & -2.409827 & 0.201065 & -0.732074\end{array}$
$\begin{array}{llll}\mathrm{H} & 2.106258 & 0.777248 & 5.063239\end{array}$
$\mathrm{H} \quad 1.174035 \quad 2.150214 \quad 4.460540$
$\begin{array}{llll}\mathrm{H} & 2.034124 & -0.243193 & 2.729930\end{array}$
$\begin{array}{llll}\mathrm{H} & 0.469207 & -0.075053 & 3.506971\end{array}$
$\mathrm{H} \quad 3.616178 \quad 2.705579 \quad 4.579664$
$\begin{array}{llll}\mathrm{H} & 6.747407 & -3.789182 & 2.313817\end{array}$
$\begin{array}{llll}\mathrm{H} & 6.456776 & -5.123859 & 1.210845\end{array}$
$\begin{array}{llll}\mathrm{H} & 4.454133 & -5.166645 & -0.100999\end{array}$
$\begin{array}{llll}\mathrm{H} & 5.724109 & -1.433952 & 1.624277\end{array}$
H $\quad 2.287435 \quad-4.132721 \quad-0.738642$
$\begin{array}{llll}\mathrm{H} & 3.578354 & -0.394172 & 0.962059\end{array}$
$\begin{array}{llll}\mathrm{H} & 1.380582 & -0.825414 & 0.205188\end{array}$
$\begin{array}{llll}\mathrm{H} & 7.969585 & -2.535610 & 0.486506\end{array}$
$\begin{array}{llll}\mathrm{H} & 0.492833 & 3.606718 & 0.684713\end{array}$
$\begin{array}{llll}\mathrm{H} & 2.733267 & 3.892703 & 0.272063\end{array}$ 

H $\quad 3.533165 \quad 4.806145 \quad-1.994391$
H $\quad 1.901627 \quad 4.560846 \quad-2.605807$
H $\quad 5.062851 \quad 2.731416 \quad-3.054008$
H $\quad 2.353526 \quad-0.416427 \quad-2.181205$
H $\quad 4.650726 \quad 0.195789 \quad-3.105648$
Н $\quad-6.316055 \quad-1.808425 \quad-4.039509$
H $\quad-5.569666 \quad-0.439627 \quad-1.372584$
H $\quad-4.888610 \quad-0.035095 \quad-2.939038$
Н $\quad-4.816228 \quad-3.818878 \quad-3.059704$
$\begin{array}{llll}\text { H } & -1.225949 & -2.709172 & -1.094585\end{array}$
H $\quad-2.501025 \quad-4.581278 \quad-2.266971$
$\mathrm{H} \quad-5.732571 \quad-2.573872 \quad 3.223947$
H $\quad-6.083908 \quad 0.309989 \quad 4.263905$
H $\quad-4.885064 \quad-0.834976 \quad 4.853332$
H $\quad-3.435885 \quad 1.460273 \quad 3.996016$
H $\quad-4.758892 \quad-1.225496 \quad 1.039162$
H $\quad-2.012538 \quad 1.858715 \quad 1.923607$
H $\quad 0.015828 \quad 4.437690 \quad-0.679627$
$\begin{array}{llll}\text { O } & -1.566950 & 1.941087 & -1.037967\end{array}$
$\begin{array}{llll}\text { O } & -1.164894 & 0.967743 & -2.023026\end{array}$ 
Table S5. Cartesian coordinates of $\mathrm{PO}^{\mathrm{T}}$ in the triplet state.
Atom $\mathrm{x}$ $\mathrm{y}$
$\mathrm{Z}$
$\begin{array}{llll}\text { C } & 2.475269 & 0.285434 & 1.245683\end{array}$
$\begin{array}{llll}\text { C } & 3.493010 & 1.177793 & 0.856402\end{array}$
$\begin{array}{llll}\text { C } & 4.678146 & 1.253841 & 1.599317\end{array}$
$\begin{array}{llll}\text { C } & 4.902385 & 0.445215 & 2.730454\end{array}$
$\begin{array}{llll}\text { C } & 3.883587 & -0.457131 & 3.085144\end{array}$
$\begin{array}{llll}\text { C } & 2.683340 & -0.539311 & 2.360939\end{array}$
C $\quad 6.154985 \quad 0.591388 \quad 3.583592$
$\begin{array}{llll}\text { C } & 7.498355 & 0.439903 & 2.837442\end{array}$
$\begin{array}{llll}\text { O } & 1.280272 & 0.208524 & 0.556165\end{array}$
$\begin{array}{llll}\text { O } & 0.591260 & 2.384468 & -0.462232\end{array}$
$\begin{array}{llll}\text { C } & 1.201584 & 2.928443 & -1.459480\end{array}$
$\begin{array}{llll}\text { O } & 2.099953 & 2.365940 & -2.134623\end{array}$
$\begin{array}{llll}\text { C } & 0.766755 & 4.345278 & -1.846079\end{array}$
$\begin{array}{llll}\text { C } & 1.944409 & 5.274511 & -2.184175\end{array}$
C $\quad 2.833673 \quad 5.570603 \quad-0.967484$
N $\quad 2.083960 \quad-0.330196 \quad-2.518312$
$\begin{array}{llll}\text { C } & 3.498577 & -0.772915 & -2.581199\end{array}$
$\begin{array}{llll}\text { C } & 3.630737 & -2.300131 & -2.703117\end{array}$
C $3.243647-3.045824-1.456982$
N $\quad 2.100612-2.747608 \quad-0.725505$
C $\quad 2.051553 \quad-3.597602 \quad 0.292899$
N $3.112319 \quad-4.437242 \quad 0.250970$
$\begin{array}{llll}\text { C } & 3.884268 & -4.102685 & -0.848699\end{array}$
$\mathrm{Cu} \quad 0.900712 \quad-1.210436 \quad-1.124971$
$\mathrm{N} \quad-2.955745 \quad 1.250256 \quad 0.296211$
C $\quad-4.248096 \quad 1.557205 \quad 0.713397$
C $\quad-4.366051 \quad 2.916135 \quad 0.898829$
$\begin{array}{llll}\mathrm{N} & -3.107580 & 3.417405 & 0.586160\end{array}$
$\begin{array}{llll}\text { C } & -2.286939 & 2.400120 & 0.228600\end{array}$
$\begin{array}{llll}\text { C } & -5.502283 & 3.802291 & 1.326185\end{array}$ 

$\begin{array}{llll}\text { C } & -6.809154 & 3.041123 & 1.594019\end{array}$
$\mathrm{Cu} \quad-2.239083 \quad-0.538781 \quad-0.116857$
$\begin{array}{llll}\mathrm{N} & -3.588421 & -1.705904 & 0.848167\end{array}$
$\begin{array}{llll}\text { C } & -4.707573 & -2.289601 & 0.255133\end{array}$
$\begin{array}{llll}\text { C } & -5.393680 & -2.986792 & 1.226931\end{array}$
$\begin{array}{llll}\mathrm{N} & -4.678778 & -2.819235 & 2.401923\end{array}$
$\begin{array}{llll}\text { C } & -3.602707 & -2.042334 & 2.133297\end{array}$
$\begin{array}{llll}\text { C } & -5.016222 & -2.126513 & -1.209488\end{array}$
$\begin{array}{llll}\text { C } & -6.377001 & -2.703705 & -1.635377\end{array}$
$\begin{array}{llll}\mathrm{H} & 2.069358 & 0.706756 & -2.330978\end{array}$
$\begin{array}{llll}\mathrm{H} & 1.649935 & -0.460195 & -3.434604\end{array}$
$\begin{array}{lllll}\mathrm{H} & 3.996322 & -0.421650 & -1.672121\end{array}$
$\begin{array}{llll}\mathrm{H} & 3.020124 & -2.648270 & -3.549518\end{array}$
$\begin{array}{llll}\mathrm{H} & 4.669060 & -2.549407 & -2.947008\end{array}$
$\begin{array}{llll}\mathrm{H} & 4.795729 & -4.624734 & -1.099068\end{array}$
$\begin{array}{llll}\mathrm{H} & 1.287737 & -3.630875 & 1.056658\end{array}$
$\begin{array}{llll}\mathrm{H} & 3.653768 & 6.247149 & -1.234564\end{array}$
$\mathrm{H} \quad 1.548379 \quad 6.215620 \quad-2.588103$
$\mathrm{H} \quad 2.540755 \quad 4.807198 \quad-2.975425$
H $\quad 0.163871 \quad 4.764926 \quad-1.031666$
H $\quad 0.114759 \quad 4.244231 \quad-2.725872$
H $\quad-6.428656 \quad-3.782929-1.451187$
$\mathrm{H} \quad-4.212434 \quad-2.601121 \quad-1.788647$
H $\quad-4.977788 \quad-1.056340 \quad-1.456950$
$\begin{array}{llll}\mathrm{H} & -6.300694 & -3.569790 & 1.181299\end{array}$
$\begin{array}{llll}\mathrm{H} & -2.877236 & -1.743193 & 2.877874\end{array}$
$\begin{array}{llll}\mathrm{H} & -6.684582 & 2.307815 & 2.398795\end{array}$
$\begin{array}{llll}\mathrm{H} & -5.674371 & 4.559662 & 0.548600\end{array}$
$\begin{array}{llll}\mathrm{H} & -5.206535 & 4.351285 & 2.231260\end{array}$
$\mathrm{H} \quad-2.831456 \quad 4.390911 \quad 0.621597$
$\begin{array}{llll}\mathrm{H} & -4.999968 & 0.794549 & 0.849627\end{array}$
$\begin{array}{llll}\mathrm{H} & -1.237262 & 2.518801 & -0.053992\end{array}$
H $\quad 7.604068 \quad 1.186339 \quad 2.042032$ 


$\begin{array}{lrrr}\mathrm{H} & 6.114733 & -0.142643 & 4.397786 \\ \mathrm{H} & 6.137282 & 1.578863 & 4.066030 \\ \mathrm{H} & 5.437838 & 1.970998 & 1.291855 \\ \mathrm{H} & 4.014992 & -1.092227 & 3.960685 \\ \mathrm{H} & 3.346260 & 1.811945 & -0.014686 \\ \mathrm{H} & 1.888975 & -1.213838 & 2.676941 \\ \mathrm{H} & 1.053867 & 1.134536 & 0.103073 \\ \mathrm{H} & 8.336943 & 0.572547 & 3.530269 \\ \mathrm{H} & 7.587154 & -0.551900 & 2.378856 \\ \mathrm{H} & 3.999988 & -0.301178 & -3.436483 \\ \mathrm{H} & 2.259962 & 6.043156 & -0.159986 \\ \mathrm{H} & 3.278224 & 4.650666 & -0.569860 \\ \mathrm{H} & -7.202270 & -2.221573 & -1.099007 \\ \mathrm{H} & -6.532903 & -2.543372 & -2.706471 \\ \mathrm{H} & -7.595587 & 3.739556 & 1.895712 \\ \mathrm{H} & -7.152587 & 2.515142 & 0.696287 \\ \mathrm{H} & 3.312859 & -5.167840 & 0.921626 \\ \mathrm{H} & -4.917972 & -3.203455 & 3.307194 \\ \mathrm{O} & -0.921104 & -0.573972 & -1.624359 \\ \mathrm{O} & -1.061620 & -1.839908 & -0.976108\end{array}$


Table S6. Cartesian coordinates of $\mathrm{PO}^{\mathrm{T}}$ in the open-shell singlet state.
Atom $\mathrm{x}$
$\mathrm{y}$
$\mathrm{Z}$
$\begin{array}{llll}\text { C } & 2.502737 & 0.290280 & 1.250415\end{array}$
$\begin{array}{llll}\text { C } & 3.488582 & 1.239824 & 0.918091\end{array}$
C $\quad 4.660943 \quad 1.324127 \quad 1.678801$
C $\quad 4.902972 \quad 0.468960 \quad 2.771226$
C $\quad 3.916630 \quad-0.486830 \quad 3.071139$
C $\quad 2.729275 \quad-0.579277 \quad 2.327956$
C $6.153823 \quad 0.610630 \quad 3.624222$
C $\quad 7.483747 \quad 0.393398 \quad 2.871318$
$\begin{array}{llll}\text { O } & 1.323978 & 0.194973 & 0.542678\end{array}$
$\begin{array}{llll}\text { O } & 0.581610 & 2.387948 & -0.443674\end{array}$
$\begin{array}{llll}\text { C } & 1.179158 & 2.940948 & -1.440964\end{array}$
$\begin{array}{llll}\text { O } & 2.063384 & 2.381295 & -2.139635\end{array}$
$\begin{array}{lllll}\text { C } & 0.753780 & 4.367950 & -1.801559\end{array}$
$\begin{array}{llll}\text { C } & 1.936355 & 5.289791 & -2.143306\end{array}$
$\begin{array}{llll}\text { C } & 2.851982 & 5.550551 & -0.938056\end{array}$
$\begin{array}{llll}\mathrm{N} & 2.100490 & -0.300326 & -2.511500\end{array}$
$\begin{array}{llll}\text { C } & 3.533909 & -0.685977 & -2.522661\end{array}$
$\begin{array}{llll}\text { C } & 3.735779 & -2.200624 & -2.691550\end{array}$
$\begin{array}{llll}\text { C } & 3.308053 & -3.008861 & -1.498694\end{array}$
$\begin{array}{llll}\mathrm{N} & 2.126477 & -2.764726 & -0.810913\end{array}$
$\begin{array}{llll}\text { C } & 2.042943 & -3.661493 & 0.164555\end{array}$
$\begin{array}{llll}\mathrm{N} & 3.120207 & -4.480010 & 0.135851\end{array}$
$\begin{array}{llll}\text { C } & 3.937984 & -4.082168 & -0.908459\end{array}$
$\mathrm{Cu} \quad 0.921020 \quad-1.234724 \quad-1.172857$
$\begin{array}{llll}\mathrm{N} & -3.007566 & 1.217845 & 0.255372\end{array}$
$\begin{array}{llll}\text { C } & -4.294353 & 1.548861 & 0.668216\end{array}$
$\begin{array}{llll}\text { C } & -4.392319 & 2.912092 & 0.831957\end{array}$
$\begin{array}{llll}\mathrm{N} & -3.127270 & 3.390374 & 0.510335\end{array}$
$\begin{array}{llll}\text { C } & -2.321014 & 2.356214 & 0.169198\end{array}$
$\begin{array}{llll}\text { C } & -5.518001 & 3.817742 & 1.244366\end{array}$ 

$\begin{array}{llll}\text { C } & -6.808381 & 3.064746 & 1.600020\end{array}$
$\mathrm{Cu} \quad-2.276372 \quad-0.573942 \quad-0.093810$
$\begin{array}{llll}\mathrm{N} & -3.618940 & -1.723015 & 0.870551\end{array}$
$\begin{array}{llll}\text { C } & -4.741352 & -2.291553 & 0.270851\end{array}$
$\begin{array}{llll}\text { C } & -5.437490 & -2.986854 & 1.236559\end{array}$
$\begin{array}{llll}\mathrm{N} & -4.723995 & -2.832697 & 2.414416\end{array}$
$\begin{array}{llll}\text { C } & -3.638904 & -2.065998 & 2.153851\end{array}$
$\begin{array}{llll}\text { C } & -5.035467 & -2.117546 & -1.194945\end{array}$
$\begin{array}{llll}\text { C } & -6.396913 & -2.678633 & -1.634653\end{array}$
H $2.045346 \quad 0.737620 \quad-2.317238$
H $\quad 1.710786 \quad-0.435572 \quad-3.446858$
$\begin{array}{llll}\mathrm{H} & 3.975891 & -0.346108 & -1.581061\end{array}$
$\begin{array}{llll}\mathrm{H} & 3.189504 & -2.540111 & -3.584277\end{array}$
$\begin{array}{llll}\mathrm{H} & 4.795747 & -2.399070 & -2.883230\end{array}$
H $4.869980 \quad-4.577161 \quad-1.137192$
H $\quad 1.241832 \quad-3.741270 \quad 0.885579$
H $3.673426 \quad 6.225061 \quad-1.206212$
$\begin{array}{llll}\mathrm{H} & 1.544638 & 6.243642 & -2.520822\end{array}$
$\mathrm{H} \quad 2.512184 \quad 4.831363 \quad-2.954683$
$\begin{array}{llll}\mathrm{H} & 0.168252 & 4.781442 & -0.971431\end{array}$
H $\quad 0.088038 \quad 4.287566 \quad-2.673224$
H $\quad-6.460887 \quad-3.758168 \quad-1.456228$
$\begin{array}{llll}\mathrm{H} & -4.231707 & -2.598293 & -1.769367\end{array}$
$\begin{array}{llll}\mathrm{H} & -4.983925 & -1.046701 & -1.436976\end{array}$
$\begin{array}{llll}\mathrm{H} & -6.349777 & -3.561070 & 1.185136\end{array}$
$\begin{array}{llll}\mathrm{H} & -2.910965 & -1.779811 & 2.901010\end{array}$
$\begin{array}{llll}\mathrm{H} & -6.649373 & 2.379374 & 2.440074\end{array}$
$\begin{array}{llll}\mathrm{H} & -5.721739 & 4.527876 & 0.430719\end{array}$
$\begin{array}{llll}\mathrm{H} & -5.195889 & 4.419345 & 2.105854\end{array}$
$\begin{array}{llll}\mathrm{H} & -2.835841 & 4.359843 & 0.532448\end{array}$
$\begin{array}{llll}\mathrm{H} & -5.056266 & 0.799088 & 0.819335\end{array}$
H $\quad-1.267063 \quad 2.457962 \quad-0.105787$
$\begin{array}{llll}\mathrm{H} & 7.597405 & 1.105788 & 2.046304\end{array}$ 

H $\quad 6.096696 \quad-0.096008 \quad 4.461214$
H $\quad 6.167766 \quad 1.613763 \quad 4.072369$
H $\quad 5.397406 \quad 2.082961 \quad 1.417537$
H $\quad 4.063640 \quad-1.157641 \quad 3.917084$
H $\quad 3.326577 \quad 1.908325 \quad 0.076178$
H $\quad 1.957225 \quad-1.297476 \quad 2.599683$
H $\quad 1.074433 \quad 1.114799 \quad 0.109061$
H $\quad 8.335633 \quad 0.527829 \quad 3.547689$
H $\quad 7.538310 \quad-0.617146 \quad 2.449686$
H $44.050849 \quad-0.163234 \quad-3.337929$
H $\quad 2.298848 \quad 6.010026 \quad-0.108991$
H $\quad 3.295058 \quad 4.617655 \quad-0.570213$
H $\quad-7.221875 \quad-2.191198 \quad-1.102615$
H $\quad-6.543007 \quad-2.511700 \quad-2.706123$
H $\quad-7.590300 \quad 3.773900 \quad 1.888163$
H $\quad-7.178477 \quad 2.486527 \quad 0.745999$
$\begin{array}{llll}\text { H } & 3.301049 & -5.238970 & 0.780168\end{array}$
H $\quad-4.969530 \quad-3.219456 \quad 3.316941$
$\begin{array}{llll}\text { O } & -0.887624 & -0.534263 & -1.482013\end{array}$
$\begin{array}{llll}\text { O } & -0.973506 & -1.817227 & -0.807427\end{array}$ 
Table S7. Cartesian coordinates of Int1a in the triplet state.
Atom $\mathrm{x} \quad \mathrm{y} \quad \mathrm{z}$
C $\quad 2.487451 \quad 0.599412 \quad 1.206326$
$\begin{array}{llll}\text { C } & 3.678241 & 0.930561 & 0.515939\end{array}$
C $\quad 4.866421 \quad 1.164774 \quad 1.214540$
C $\quad 4.930050 \quad 1.079573 \quad 2.620461$
C $\quad 3.743014 \quad 0.748534 \quad 3.299324$
$\begin{array}{llll}\text { C } & 2.540973 & 0.511867 & 2.613003\end{array}$
C $\quad 6.217929 \quad 1.378643 \quad 3.369161$
C $\quad 7.376139 \quad 0.400677 \quad 3.075311$
$\begin{array}{llll}\text { O } & 1.318784 & 0.391309 & 0.547012\end{array}$
$\begin{array}{llll}\text { O } & 0.851004 & 2.474792 & -0.874309\end{array}$
C $\quad 1.322226 \quad 2.347421 \quad-2.114756$
O $\quad 1.838589 \quad 1.308026-2.530788$
C $\quad 1.201399 \quad 3.601074 \quad-2.958708$
C $\quad 2.554587 \quad 4.346533 \quad-3.070298$
$\begin{array}{llll}\text { C } & 3.050190 & 4.942410 & -1.743399\end{array}$
$\mathrm{N} \quad 1.170498 \quad-1.556634 \quad-2.830699$
C $\quad 2.102382 \quad-2.600760 \quad-3.308408$
C $\quad 2.068971 \quad-3.821305 \quad-2.374300$
$\begin{array}{llll}\text { C } & 2.695529 & -3.556902 & -1.034444\end{array}$
N $\quad 2.426527 \quad-2.419589 \quad-0.278675$
$\begin{array}{llll}\text { C } & 3.145062 & -2.514898 & 0.835730\end{array}$
N $\quad 3.861045 \quad-3.663372 \quad 0.833541$
C $\quad 3.595960 \quad-4.338848 \quad-0.346256$
$\begin{array}{llll}\mathrm{Cu} & 1.131838 & -0.955283 & -0.848286\end{array}$
$\begin{array}{llll}\mathrm{N} & -2.869837 & 1.414990 & 0.415774\end{array}$
$\begin{array}{llll}\text { C } & -4.151398 & 1.801085 & 0.787459\end{array}$
$\begin{array}{llll}\text { C } & -4.235894 & 3.175721 & 0.828418\end{array}$
$\begin{array}{llll}\mathrm{N} & -2.963100 & 3.609213 & 0.478330\end{array}$
$\begin{array}{llll}\text { C } & -2.173945 & 2.532110 & 0.234224\end{array}$
$\begin{array}{llll}\text { C } & -5.357238 & 4.123728 & 1.151344\end{array}$ 


$$
\begin{array}{cccc}
\mathrm{C} & -6.747091 & 3.474707 & 1.050024 \\
\mathrm{Cu} & -2.294226 & -0.437254 & -0.032847 \\
\mathrm{~N} & -3.582292 & -1.573115 & 1.055783 \\
\mathrm{C} & -4.743109 & -2.214872 & 0.631089 \\
\mathrm{C} & -5.265359 & -2.916766 & 1.697479 \\
\mathrm{~N} & -4.408625 & -2.694375 & 2.763029 \\
\mathrm{C} & -3.412042 & -1.881704 & 2.337036 \\
\mathrm{C} & -5.257122 & -2.100066 & -0.778117 \\
\mathrm{C} & -6.606551 & -2.800247 & -1.005289 \\
\mathrm{H} & 1.349318 & -0.656277 & -3.282730 \\
\mathrm{H} & 0.202028 & -1.816005 & -3.024398 \\
\mathrm{H} & 3.110938 & -2.174952 & -3.333821 \\
\mathrm{H} & 1.024872 & -4.140798 & -2.245951 \\
\mathrm{H} & 2.598308 & -4.654499 & -2.848763 \\
\mathrm{H} & 4.054862 & -5.284841 & -0.592372 \\
\mathrm{H} & 3.178524 & -1.781015 & 1.629898 \\
\mathrm{H} & 3.990894 & 5.483935 & -1.893923 \\
\mathrm{H} & 2.430910 & 5.149325 & -3.807689 \\
\mathrm{H} & 3.303065 & 3.656467 & -3.478654 \\
\mathrm{H} & 0.444343 & 4.263512 & -2.525601 \\
\mathrm{H} & 0.875290 & 3.292869 & -3.958097 \\
\mathrm{H} & -6.535622 & -3.876590 & -0.810663 \\
\mathrm{H} & -4.499599 & -2.510387 & -1.459121 \\
\mathrm{H} & -5.346108 & -1.034629 & -1.032585 \\
\mathrm{H} & -6.144712 & -3.536217 & 1.783609 \\
\mathrm{H} & -2.602778 & -1.543969 & 2.970492 \\
\mathrm{H} & -6.847815 & 2.638041 & 1.750554 \\
\mathrm{H} & -5.212994 & 4.528521 & 2.162815 \\
\mathrm{H} & -2.664693 & 4.574211 & 0.410238 \\
\mathrm{H} & -1.074426 & 0.992578 \\
\mathrm{H} & 2.598245 & -0.083382 \\
\mathrm{H} & 0.390464 & 2.008910
\end{array}
$$



$\begin{array}{llll}\mathrm{H} & 6.015283 & 1.380175 & 4.446969\end{array}$
$\begin{array}{llll}\mathrm{H} & 6.549818 & 2.395381 & 3.118085\end{array}$
$\begin{array}{llll}\mathrm{H} & 5.760679 & 1.433267 & 0.652843\end{array}$
H $\quad 3.750312 \quad 0.688597 \quad 4.387298$
H $\quad 3.653497 \quad 1.002969 \quad-0.569964$
$\mathrm{H} \quad 1.627245 \quad 0.274730 \quad 3.155134$
H $\quad 1.044266 \quad 1.621887 \quad-0.324737$
$\begin{array}{llll}\mathrm{H} & 8.276038 & 0.690522 & 3.629688\end{array}$
H $\quad 7.112143 \quad-0.622730 \quad 3.367631$
H $\quad 1.847133 \quad-2.915043 \quad-4.329431$
$\mathrm{H} \quad 2.319514 \quad 5.648308 \quad-1.329922$
H $\quad 3.226541 \quad 4.167673 \quad-0.988692$
H $\quad-7.387831 \quad-2.383574 \quad-0.359106$
H $\quad-6.927293 \quad-2.672429 \quad-2.043788$
$\begin{array}{llll}\mathrm{H} & -7.523342 & 4.206298 & 1.295005\end{array}$
H $\quad-6.935826 \quad 3.101735 \quad 0.037759$
$\mathrm{H} \quad 4.499339 \quad-3.955141 \quad 1.562345$
H $\quad-4.506431 \quad-3.069053 \quad 3.697800$
$\begin{array}{llll}\mathrm{O} & -0.723555 & -0.365816 & -1.304776\end{array}$
$\begin{array}{lllll}\text { O } & -1.392449 & -1.623930 & -1.225400\end{array}$ 
Table S8. Cartesian coordinates of Int1a in the open-shell singlet state.
Atom $\mathrm{x} \quad \mathrm{y} \quad \mathrm{z}$
C $\quad 2.515581 \quad 0.624602 \quad 1.206700$
C $\quad 3.695097 \quad 0.999811 \quad 0.520076$
C $\quad 4.878245 \quad 1.249782 \quad 1.221447$
C $\quad 4.945705 \quad 1.137462 \quad 2.625003$
C $\quad 3.769089 \quad 0.765965 \quad 3.300705$
C $\quad 2.572667 \quad 0.513009 \quad 2.611563$
C $\quad 6.236476 \quad 1.424318 \quad 3.370242$
C $\quad 7.343770 \quad 0.375886 \quad 3.129744$
$\begin{array}{llll}\text { O } & 1.353309 & 0.394505 & 0.546428\end{array}$
O $\quad 0.852444 \quad 2.451990 \quad-0.886406$
C $\quad 1.312191 \quad 2.303236-2.128041$
O $\quad 1.809218 \quad 1.250648 \quad-2.535993$
$\begin{array}{llll}\text { C } & 1.207890 & 3.549297 & -2.985310\end{array}$
$\begin{array}{llll}\text { C } & 2.571505 & 4.275259 & -3.101976\end{array}$
C $\quad 3.075522 \quad 4.874760 \quad-1.779771$
$\mathrm{N} \quad 1.073156 \quad-1.603166 \quad-2.792629$
C $\quad 1.996934 \quad-2.634974 \quad-3.310985$
C $\quad 2.058862 \quad-3.835511 \quad-2.353249$
C $\quad 2.763191 \quad-3.527348 \quad-1.062286$
$\mathrm{N} \quad 2.514525 \quad-2.381109 \quad-0.312829$
$\begin{array}{llll}\text { C } & 3.298652 & -2.440068 & 0.759285\end{array}$
$\begin{array}{llll}\mathrm{N} & 4.038467 & -3.572773 & 0.734862\end{array}$
C $3.719649 \quad-4.276452 \quad-0.414664$
$\begin{array}{llll}\mathrm{Cu} & 1.152631 & -0.968676 & -0.833592\end{array}$
$\begin{array}{llll}\mathrm{N} & -2.899742 & 1.395477 & 0.385585\end{array}$
$\begin{array}{llll}\text { C } & -4.178153 & 1.807875 & 0.737901\end{array}$
$\begin{array}{llll}\text { C } & -4.242824 & 3.184047 & 0.745817\end{array}$
$\begin{array}{llll}\mathrm{N} & -2.961471 & 3.591231 & 0.395422\end{array}$
$\begin{array}{llll}\text { C } & -2.186116 & 2.497680 & 0.183084\end{array}$
$\begin{array}{llll}\text { C } & -5.353992 & 4.153592 & 1.036169\end{array}$ 

$\begin{array}{llll}\text { C } & -6.747396 & 3.508276 & 0.974689\end{array}$
$\begin{array}{llll}\mathrm{Cu} & -2.324972 & -0.470232 & 0.003565\end{array}$
$\begin{array}{llll}\mathrm{N} & -3.643191 & -1.548256 & 1.100018\end{array}$
$\begin{array}{llll}\text { C } & -4.801692 & -2.188683 & 0.669800\end{array}$
$\begin{array}{llll}\text { C } & -5.348459 & -2.862160 & 1.742108\end{array}$
$\begin{array}{llll}\mathrm{N} & -4.507486 & -2.623666 & 2.816813\end{array}$
$\begin{array}{llll}\text { C } & -3.496190 & -1.829835 & 2.390324\end{array}$
$\begin{array}{llll}\text { C } & -5.285692 & -2.100210 & -0.751262\end{array}$
$\begin{array}{llll}\text { C } & -6.634096 & -2.796300 & -0.989641\end{array}$
H $\quad \begin{array}{lll}1.198536 & -0.711282 & -3.277461\end{array}$
$\begin{array}{lllll}\mathrm{H} & 0.098666 & -1.889377 & -2.905338\end{array}$
$\begin{array}{llll}\mathrm{H} & 2.989795 & -2.184786 & -3.414431\end{array}$
$\begin{array}{llll}\mathrm{H} & 1.034821 & -4.179941 & -2.149717\end{array}$
$\begin{array}{llll}\mathrm{H} & 2.578985 & -4.664063 & -2.845629\end{array}$
H $4.183499 \quad-5.217544 \quad-0.670027$
$\begin{array}{llll}\mathrm{H} & 3.357703 & -1.693747 & 1.539934\end{array}$
$\mathrm{H} \quad 4.023580 \quad 5.401994 \quad-1.934972$
$\mathrm{H} \quad 2.459190 \quad 5.073777 \quad-3.845801$
$\mathrm{H} \quad 3.310304 \quad 3.571571 \quad-3.504762$
$\begin{array}{lllll}\mathrm{H} & 0.459800 & 4.226377 & -2.559218\end{array}$
H $\quad 0.879068 \quad 3.236122 \quad-3.982226$
H $\quad-6.573990 \quad-3.868559-0.770406$
$\begin{array}{llll}\mathrm{H} & -4.517228 & -2.530678 & -1.407253\end{array}$
$\begin{array}{lllll}\mathrm{H} & -5.362133 & -1.039845 & -1.030027\end{array}$
$\begin{array}{llll}\mathrm{H} & -6.234433 & -3.472270 & 1.826885\end{array}$
$\mathrm{H} \quad-2.693211 \quad-1.487002 \quad 3.028960$
$\mathrm{H} \quad-6.846045 \quad 2.703313 \quad 1.711699$
$\begin{array}{llll}\mathrm{H} & -5.302690 & 4.979140 & 0.313157\end{array}$
$\begin{array}{llll}\mathrm{H} & -5.198562 & 4.601078 & 2.027818\end{array}$
$\begin{array}{llll}\mathrm{H} & -2.648236 & 4.549911 & 0.307446\end{array}$
H $\quad-4.962088 \quad 1.097522 \quad 0.954731$
$\begin{array}{llll}\mathrm{H} & -1.148927 & 2.542145 & -0.126616\end{array}$
$\begin{array}{llll}\mathrm{H} & 7.596261 & 0.302798 & 2.065579\end{array}$ 

H $\quad 6.029126 \quad 1.487463 \quad 4.445250$
H $\quad 6.616995 \quad 2.409767 \quad 3.070025$
H $\quad 5.766347 \quad 1.548981 \quad 0.665200$
H $\quad 3.781079 \quad 0.684552 \quad 4.387240$
H $\quad 3.666941 \quad 1.087105 \quad-0.564560$
H $\quad 1.666017 \quad 0.240524 \quad 3.148769$
H $1.051936 \quad 1.604993 \quad-0.324845$
$\begin{array}{llll}\text { H } & 8.257001 & 0.643431 & 3.673697\end{array}$
H $\quad 7.025538 \quad-0.617141 \quad 3.469378$
H $\quad 1.679915 \quad-2.976767 \quad-4.305470$
H $\quad 2.354831 \quad 5.594122 \quad-1.372057$
Н $\quad 3.241029 \quad 4.104185 \quad-1.018491$
H $\quad-7.425062 \quad-2.361061 \quad-0.367906$
H $\quad-6.934432 \quad-2.689382 \quad-2.036599$
H $\quad-7.518270 \quad 4.253575 \quad 1.193949$
H $\quad-6.946714 \quad 3.092889 \quad-0.018892$
H $\quad 4.723283 \quad-3.837515 \quad 1.431034$
H $\quad-4.623563 \quad-2.977717 \quad 3.757532$
$\begin{array}{llll}\text { O } & -0.723792 & -0.416696 & -1.167267\end{array}$
$\begin{array}{llll}\mathrm{O} & -1.355560 & -1.709748 & -1.068051\end{array}$ 
Table S9. Cartesian coordinates of Int1b in the triplet state. 


$$
\begin{array}{cccc}
\mathrm{C} & -6.981448 & 1.581201 & 3.060744 \\
\mathrm{Cu} & -2.345634 & -0.471613 & -0.377996 \\
\mathrm{~N} & -2.851854 & -2.165079 & 0.562623 \\
\mathrm{C} & -3.952770 & -3.001999 & 0.375119 \\
\mathrm{C} & -3.806649 & -4.092743 & 1.205209 \\
\mathrm{~N} & -2.612645 & -3.906275 & 1.885096 \\
\mathrm{C} & -2.065938 & -2.740112 & 1.468471 \\
\mathrm{C} & -5.039719 & -2.694711 & -0.622930 \\
\mathrm{C} & -6.141362 & -3.765594 & -0.724055 \\
\mathrm{H} & 1.392151 & 1.278861 & -2.520519 \\
\mathrm{H} & 0.612977 & -0.011747 & -3.158761 \\
\mathrm{H} & 3.491607 & 0.600201 & -3.298174 \\
\mathrm{H} & 2.024032 & -1.969968 & -4.106850 \\
\mathrm{H} & 3.627070 & -1.550724 & -4.700064 \\
\mathrm{H} & 5.343433 & -3.244776 & -3.306746 \\
\mathrm{H} & 3.805202 & -2.464395 & 0.568906 \\
\mathrm{H} & -3.383872 & 2.339321 & -1.439844 \\
\mathrm{H} & 3.140758 & 5.893813 & 0.374680 \\
\mathrm{H} & 1.298655 & 6.819053 & -1.042781 \\
\mathrm{H} & 2.085087 & 5.472038 & -1.865361 \\
\mathrm{H} & -0.540495 & 5.214812 & -0.276824 \\
\mathrm{H} & -0.444842 & 5.447235 & -2.018684 \\
\mathrm{H} & -5.729612 & -4.734989 & -1.026882 \\
\mathrm{H} & -4.570064 & -2.560623 & -1.607376 \\
\mathrm{H} & -5.489864 & -1.726005 & -0.369158 \\
\mathrm{H} & -4.427679 & -4.962181 & 1.356983 \\
\mathrm{H} & -6.11934918 & -2.351000 & 1.815394 \\
\mathrm{H} & 1.519617 & 3.734488 \\
\mathrm{H} & -56526 & 2.534378 & 1.204717 \\
\mathrm{H} & 3.476413 & 2.170838 \\
\mathrm{H} & -3.592250 & -0.353396 \\
\mathrm{H} & -0.451266
\end{array}
$$



$\mathrm{H} \quad 6.180644 \quad 0.027789 \quad 4.974254$
$\begin{array}{llll}\mathrm{H} & 6.088520 & 1.670474 & 4.362414\end{array}$
$\mathrm{H} \quad 4.483061 \quad-1.521815 \quad 4.352876$
$\mathrm{H} \quad 5.127060 \quad 1.936957 \quad 1.868210$
H $\quad 2.462509 \quad-2.095470 \quad 3.029355$
H $\quad 3.102014 \quad 1.384024 \quad 0.567159$
$\begin{array}{llll}\mathrm{H} & -0.842127 & 1.890160 & -1.218235\end{array}$
$\begin{array}{llll}\mathrm{H} & 8.371362 & 0.769620 & 4.037527\end{array}$
H $7.744595 \quad-0.595444 \quad 3.091272$
H $\quad 2.370343 \quad 0.494933 \quad-4.662471$
$\begin{array}{llll}\mathrm{H} & 1.582505 & 5.638843 & 1.176292\end{array}$
H $\quad 2.388896 \quad 4.291846 \quad 0.349289$
$\begin{array}{llll}\mathrm{H} & -6.663346 & -3.895919 & 0.231104\end{array}$
H $\quad-6.882882 \quad-3.469062-1.472229$
$\begin{array}{llll}\mathrm{H} & -7.819488 & 2.007081 & 3.620642\end{array}$
$\begin{array}{llll}\mathrm{H} & -7.260844 & 0.564301 & 2.762900\end{array}$
H $\quad 5.597960 \quad-3.668642 \quad-0.785723$
H $\quad-2.210503 \quad-4.532489 \quad 2.570551$
$\begin{array}{llll}\text { O } & -1.219487 & 0.408748 & -1.679908\end{array}$
$\begin{array}{llll}\text { O } & -0.558297 & -0.758330 & -1.141319\end{array}$ 
Table S10. Cartesian coordinates of Int1b in the open-shell singlet state.
Atom $\quad \mathrm{x} \quad \mathrm{y} \quad \mathrm{z}$
C $\quad 2.800941 \quad-0.781900 \quad 1.635297$
C $\quad 3.419320 \quad-1.697378 \quad 2.526932$
C $\quad 4.507969-1.310190 \quad 3.322331$
C $\quad 5.053521 \quad-0.013338 \quad 3.260697$
C $\quad 4.445944 \quad 0.891939 \quad 2.364648$
C $\quad 3.348489 \quad 0.528817 \quad 1.577685$
C $\quad 6.201498 \quad 0.411203 \quad 4.164952$
C $\quad 7.572978 \quad 0.591385 \quad 3.470099$
O $\quad 1.744419 \quad-1.156862 \quad 0.904447$
O $\quad-1.145251 \quad 3.079837 \quad-0.768675$
C $\quad-0.042236 \quad 3.720541 \quad-1.145499$
O $\quad 0.915862 \quad 3.158901 \quad-1.680770$
C $\quad-0.092856 \quad 5.215546-0.882420$
C $\quad 1.291372 \quad 5.865905 \quad-0.747875$
$\begin{array}{llll}\text { C } & 2.045482 & 5.413028 & 0.510931\end{array}$
$\begin{array}{llll}\mathrm{N} & 1.301765 & 0.482947 & -2.604215\end{array}$
$\begin{array}{llll}\text { C } & 2.302810 & 0.458007 & -3.694741\end{array}$
C $\quad 2.727239 \quad-0.976471 \quad-4.048667$
C $\quad 3.538043 \quad-1.626084 \quad-2.964754$
N $\quad 3.179756 \quad-1.554662 \quad-1.624511$
C $\quad 4.099030 \quad-2.220123 \quad-0.933859$
N $\quad 5.035128 \quad-2.720398 \quad-1.773492$
$\begin{array}{llll}\text { C } & 4.702195 & -2.354287 & -3.067962\end{array}$
$\begin{array}{llll}\mathrm{Cu} & 1.556769 & -0.592101 & -0.900098\end{array}$
$\begin{array}{llll}\mathrm{N} & -3.984251 & 0.681734 & -0.022383\end{array}$
$\begin{array}{llll}\text { C } & -4.886333 & 0.606424 & 1.032117\end{array}$
$\begin{array}{llll}\text { C } & -5.697579 & 1.720833 & 1.038347\end{array}$
$\begin{array}{llll}\mathrm{N} & -5.263097 & 2.470348 & -0.048750\end{array}$
$\begin{array}{llll}\text { C } & -4.236537 & 1.822201 & -0.652294\end{array}$
$\begin{array}{llll}\text { C } & -6.816762 & 2.175037 & 1.933147\end{array}$ 


$$
\begin{aligned}
& \begin{array}{llll}
\text { C } & -7.002277 & 1.293646 & 3.178002
\end{array} \\
& \mathrm{Cu} \quad-2.360364 \quad-0.457039 \quad-0.307981 \\
& \begin{array}{llll}
\mathrm{N} & -2.701276 & -2.264557 & 0.444551
\end{array} \\
& \begin{array}{llll}
\text { C } & -3.766689 & -3.149604 & 0.266787
\end{array} \\
& \begin{array}{llll}
\text { C } & -3.473325 & -4.310014 & 0.949774
\end{array} \\
& \begin{array}{llll}
\mathrm{N} & -2.228183 & -4.115890 & 1.529877
\end{array} \\
& \begin{array}{llll}
\text { C } & -1.792743 & -2.878555 & 1.200262
\end{array} \\
& \begin{array}{llll}
\text { C } & -4.964273 & -2.810202 & -0.584435
\end{array} \\
& \begin{array}{llll}
\text { C } & -6.002130 & -3.938679 & -0.726036
\end{array} \\
& \begin{array}{llll}
\mathrm{H} & 1.161540 & 1.456478 & -2.277845
\end{array} \\
& \begin{array}{lllll}
\mathrm{H} & 0.380505 & 0.195161 & -2.943324
\end{array} \\
& \begin{array}{llll}
\mathrm{H} & 3.174445 & 1.035392 & -3.369255
\end{array} \\
& \text { H } \quad \begin{array}{llll}
1.828390 & -1.574250 & -4.260479
\end{array} \\
& \begin{array}{llll}
\mathrm{H} & 3.317333 & -0.960904 & -4.971124
\end{array} \\
& \begin{array}{llll}
\mathrm{H} & 5.300617 & -2.629485 & -3.923690
\end{array} \\
& \begin{array}{llll}
\mathrm{H} & 4.123488 & -2.327523 & 0.142839
\end{array} \\
& \begin{array}{llll}
\mathrm{H} & 3.018024 & 5.912741 & 0.584229
\end{array} \\
& \mathrm{H} \quad 1.159147 \quad 6.954981 \quad-0.726194 \\
& \begin{array}{lllll}
\mathrm{H} & 1.883374 & 5.631492 & -1.639626
\end{array} \\
& \begin{array}{llll}
\mathrm{H} & -0.707713 & 5.386492 & 0.008451
\end{array} \\
& \begin{array}{lllll}
\mathrm{H} & -0.640866 & 5.654985 & -1.728839
\end{array} \\
& \begin{array}{llll}
\mathrm{H} & -5.563617 & -4.830542 & -1.187826
\end{array} \\
& \begin{array}{llll}
\mathrm{H} & -4.602205 & -2.522071 & -1.581199
\end{array} \\
& \begin{array}{llll}
\mathrm{H} & -5.449437 & -1.916282 & -0.172758
\end{array} \\
& \begin{array}{llll}
\mathrm{H} & -4.024407 & -5.231293 & 1.060427
\end{array} \\
& \begin{array}{llll}
\mathrm{H} & -0.833489 & -2.465296 & 1.492332
\end{array} \\
& \begin{array}{llll}
\mathrm{H} & -6.097758 & 1.284974 & 3.795784
\end{array} \\
& \begin{array}{llll}
\mathrm{H} & -7.752137 & 2.198062 & 1.356411
\end{array} \\
& \begin{array}{llll}
\mathrm{H} & -6.620724 & 3.209966 & 2.246054
\end{array} \\
& \begin{array}{llll}
\mathrm{H} & -5.635091 & 3.367619 & -0.334580
\end{array} \\
& \begin{array}{llll}
\mathrm{H} & -4.878867 & -0.221598 & 1.725744
\end{array} \\
& \begin{array}{llll}
\mathrm{H} & -3.695947 & 2.209646 & -1.504091
\end{array} \\
& \text { H } \quad 7.520832 \quad 1.349254 \quad 2.679601
\end{aligned}
$$




$\begin{array}{cccc}\mathrm{H} & 6.310226 & -0.326403 & 4.969636 \\ \mathrm{H} & 5.936421 & 1.358780 & 4.653105 \\ \mathrm{H} & 4.935380 & -2.034158 & 4.015998 \\ \mathrm{H} & 4.822883 & 1.912534 & 2.298717 \\ \mathrm{H} & 3.005485 & -2.701801 & 2.606163 \\ \mathrm{H} & 2.880085 & 1.264315 & 0.923049 \\ \mathrm{H} & -1.077788 & 2.079957 & -0.986885 \\ \mathrm{H} & 8.330272 & 0.910421 & 4.195331 \\ \mathrm{H} & 7.910537 & -0.346454 & 3.013443 \\ \mathrm{H} & 1.898134 & 0.948818 & -4.589838 \\ \mathrm{H} & 1.479315 & 5.650571 & 1.420254 \\ \mathrm{H} & 2.226081 & 4.332277 & 0.494878 \\ \mathrm{H} & -6.419572 & -4.224391 & 0.246490 \\ \mathrm{H} & -6.829947 & -3.606318 & -1.359964 \\ \mathrm{H} & -7.825677 & 1.676106 & 3.788698 \\ \mathrm{H} & -7.243226 & 0.260763 & 2.902403 \\ \mathrm{H} & 5.849284 & -3.250940 & -1.491172 \\ \mathrm{H} & -1.722195 & -4.782652 & 2.098578 \\ \mathrm{O} & -1.178032 & 0.590251 & -1.414668 \\ \mathrm{O} & -0.412539 & -0.248018 & -0.520830\end{array}$


Table S11. Cartesian coordinates of TS1 in the triplet state.
Atom $\mathrm{x}$
$\mathrm{y}$
$\mathrm{Z}$
C $\quad 3.015289 \quad-0.728329 \quad 2.111818$
C $\quad 4.105963 \quad-1.291997 \quad 2.893050$
C $\quad 5.077844 \quad-0.483812 \quad 3.451726$
$\begin{array}{llll}\text { C } & 5.062409 & 0.930040 & 3.273880\end{array}$
C $\quad 4.017136 \quad 1.496064 \quad 2.494742$
C $\quad 3.022710 \quad 0.713109 \quad 1.931409$
C $\quad 6.100765 \quad 1.805287 \quad 3.942827$
C $\quad 7.561965 \quad 1.547084 \quad 3.501755$
O $\quad 2.098705 \quad-1.462370 \quad 1.619642$
O $\quad-1.075093 \quad 3.056468 \quad-1.255655$
C $\quad 0.027384 \quad 3.647757 \quad-1.709775$
O $\quad 1.017946 \quad 3.033507 \quad-2.103372$
$\begin{array}{llll}\text { C } & -0.071749 & 5.166271 & -1.724948\end{array}$
C $\quad 1.281682 \quad 5.869944 \quad-1.550964$
$\begin{array}{llll}\text { C } & 1.884287 & 5.666347 & -0.152802\end{array}$
$\begin{array}{llll}\mathrm{N} & 1.500405 & 0.248210 & -2.954204\end{array}$
$\begin{array}{llll}\text { C } & 2.682753 & -0.034589 & -3.790643\end{array}$
C $\quad 2.851481 \quad-1.548721 \quad-4.039408$
$\begin{array}{llll}\text { C } & 3.287704 & -2.341562 & -2.838359\end{array}$
N $\quad 2.634133 \quad-2.292146 \quad-1.609746$
$\begin{array}{llll}\text { C } & 3.276610 & -3.117099 & -0.791424\end{array}$
N $\quad 4.317063 \quad-3.702459 \quad-1.436527$
C $\quad 4.342400 \quad-3.222963 \quad-2.736121$
$\begin{array}{llll}\mathrm{Cu} & 1.198570 & -1.018968 & -1.232663\end{array}$
$\begin{array}{llll}\mathrm{N} & -3.926120 & 0.766263 & -0.007309\end{array}$
$\begin{array}{llll}\text { C } & -4.866073 & 0.772464 & 1.014793\end{array}$
$\begin{array}{llll}\text { C } & -5.656320 & 1.898142 & 0.923442\end{array}$
$\begin{array}{llll}\mathrm{N} & -5.169793 & 2.572017 & -0.190444\end{array}$
$\begin{array}{llll}\text { C } & -4.134141 & 1.869272 & -0.714950\end{array}$
C $\quad-6.798024 \quad 2.420620 \quad 1.749482$ 


$$
\begin{array}{cccc}
\mathrm{C} & -7.025769 & 1.624318 & 3.043432 \\
\mathrm{Cu} & -2.306361 & -0.409796 & -0.212957 \\
\mathrm{~N} & -2.528349 & -2.142143 & 0.752402 \\
\mathrm{C} & -3.555214 & -3.084978 & 0.729105 \\
\mathrm{C} & -3.187466 & -4.140104 & 1.536365 \\
\mathrm{~N} & -1.932868 & -3.824743 & 2.037204 \\
\mathrm{C} & -1.567233 & -2.619996 & 1.539424 \\
\mathrm{C} & -4.792193 & -2.903283 & -0.111788 \\
\mathrm{C} & -5.785702 & -4.075766 & -0.053276 \\
\mathrm{H} & 1.471805 & 1.230101 & -2.651245 \\
\mathrm{H} & 0.643561 & 0.111500 & -3.492488 \\
\mathrm{H} & 3.567505 & 0.353925 & -3.273602 \\
\mathrm{H} & 1.900594 & -1.949119 & -4.421090 \\
\mathrm{H} & 3.591945 & -1.697657 & -4.833370 \\
\mathrm{H} & 5.082876 & -3.537324 & -3.456551 \\
\mathrm{H} & 3.025241 & -3.277832 & 0.247831 \\
\mathrm{H} & 2.837086 & 6.199534 & -0.055952 \\
\mathrm{H} & 1.142879 & 6.942119 & -1.739170 \\
\mathrm{H} & 1.976323 & 5.495663 & -2.311120 \\
\mathrm{H} & -0.788197 & 5.476633 & -0.956258 \\
\mathrm{H} & -0.514366 & 5.433032 & -2.695668 \\
\mathrm{H} & -5.325354 & -5.007192 & -0.402164 \\
\mathrm{H} & -4.475923 & -2.743575 & -1.151922 \\
\mathrm{H} & -5.296615 & -1.977282 & 0.192161 \\
\mathrm{H} & -3.691549 & -5.062340 & 1.781108 \\
\mathrm{H} & -0.617208 & -2.138868 & 1.742262 \\
\mathrm{H} & -6.139105 & 1.651233 & 3.686125 \\
\mathrm{H} & -5.603370 & 3.472918 & 1.999212 \\
\mathrm{H} & -3.4539 & 3.455984 & -0.544256 \\
\mathrm{H} & -0.010387 & 1.758387 \\
\mathrm{H} & 2.196419 & -1.564765 \\
\mathrm{H} & -1.735008 & 2.430175
\end{array}
$$



$\begin{array}{llll}\mathrm{H} & 6.036026 & 1.648020 & 5.029263\end{array}$
$\mathrm{H} \quad 5.853105 \quad 2.857950 \quad 3.766324$
H $\quad 5.866970 \quad-0.930798 \quad 4.054673$
H $3.995894 \quad 2.575006 \quad 2.349759$
$\mathrm{H} \quad 4.109224 \quad-2.369323 \quad 3.047573$
H $\quad 2.217114 \quad 1.148098 \quad 1.343302$
$\begin{array}{llll}\mathrm{H} & -0.997839 & 2.027665 & -1.298298\end{array}$
$\begin{array}{llll}\mathrm{H} & 8.239222 & 2.210739 & 4.049508\end{array}$
H $\quad 7.868233 \quad 0.514581 \quad 3.703249$
H $\quad 2.624924 \quad 0.473526 \quad-4.764603$
H $\quad 1.210965 \quad 6.040659 \quad 0.628695$
$\begin{array}{llll}\mathrm{H} & 2.074213 & 4.603952 & 0.039983\end{array}$
H $\quad-6.157127 \quad-4.236554 \quad 0.965613$

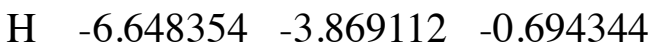
$\begin{array}{llll}\mathrm{H} & -7.863856 & 2.049689 & 3.603883\end{array}$
$\begin{array}{llll}\mathrm{H} & -7.265406 & 0.576806 & 2.828794\end{array}$
H $\quad 4.961888 \quad-4.368769-1.032770$
$\begin{array}{llll}\mathrm{H} & -1.377367 & -4.393373 & 2.662792\end{array}$
$\begin{array}{llll}\text { O } & -1.147955 & 0.520215 & -1.463838\end{array}$
$\begin{array}{llll}\mathrm{O} & -0.347444 & -0.180797 & -0.480530\end{array}$ 
Table S12. Cartesian coordinates of TS1 in the open-shell singlet state.
Atom $\quad \mathrm{x} \quad \mathrm{y} \quad \mathrm{z}$
$\begin{array}{llll}\text { C } & 3.007448 & -0.707991 & 2.101617\end{array}$
$\begin{array}{llll}\text { C } & 4.094435 & -1.283238 & 2.879231\end{array}$
$\begin{array}{llll}\text { C } & 5.069849 & -0.484363 & 3.445469\end{array}$
$\begin{array}{llll}\text { C } & 5.062339 & 0.930642 & 3.277798\end{array}$
$\begin{array}{llll}\text { C } & 4.021584 & 1.507847 & 2.500801\end{array}$
C $\quad 3.023868 \quad 0.734292 \quad 1.930408$
C $\quad 6.104702 \quad 1.795572 \quad 3.953942$
C $\quad 7.564472 \quad 1.535332 \quad 3.509417$
$\begin{array}{llll}\text { O } & 2.085556 & -1.432943 & 1.605372\end{array}$
$\begin{array}{llll}\mathrm{O} & -1.073370 & 3.052660 & -1.247127\end{array}$
C $\quad 0.027925 \quad 3.644763 \quad-1.704212$
$\begin{array}{llll}\mathrm{O} & 1.016558 & 3.030824 & -2.102371\end{array}$
$\begin{array}{llll}\text { C } & -0.071175 & 5.163251 & -1.715769\end{array}$
C $\quad 1.283568 \quad 5.866152-1.549136$
C $\quad 1.896028 \quad 5.657636 \quad-0.156028$
$\begin{array}{llll}\mathrm{N} & 1.506742 & 0.244109 & -2.958248\end{array}$
$\begin{array}{llll}\text { C } & 2.692713 & -0.036957 & -3.789856\end{array}$
$\begin{array}{llll}\text { C } & 2.865749 & -1.551078 & -4.036607\end{array}$
$\begin{array}{llll}\text { C } & 3.301586 & -2.341637 & -2.833980\end{array}$
N $\quad 2.642893 \quad-2.296086 \quad-1.608013$
$\begin{array}{llll}\text { C } & 3.287249 & -3.117008 & -0.787385\end{array}$
$\begin{array}{llll}\mathrm{N} & 4.333910 & -3.696327 & -1.428266\end{array}$
$\begin{array}{llll}\text { C } & 4.361260 & -3.216690 & -2.727742\end{array}$
$\mathrm{Cu} \quad 1.200592 \quad-1.030256 \quad-1.235575$
$\begin{array}{llll}\mathrm{N} & -3.930529 & 0.760878 & -0.017223\end{array}$
$\begin{array}{llll}\text { C } & -4.867705 & 0.771348 & 1.007427\end{array}$
$\begin{array}{llll}\text { C } & -5.660788 & 1.894546 & 0.910898\end{array}$
$\begin{array}{llll}\mathrm{N} & -5.178804 & 2.562376 & -0.208661\end{array}$
$\begin{array}{llll}\text { C } & -4.142906 & 1.858864 & -0.731408\end{array}$
$\begin{array}{llll}\text { C } & -6.801653 & 2.419533 & 1.736574\end{array}$ 


$$
\begin{aligned}
& \begin{array}{llll}
\text { C } & -7.022708 & 1.632642 & 3.037494
\end{array} \\
& \mathrm{Cu} \quad-2.308355 \quad-0.409815 \quad-0.214919 \\
& \begin{array}{llll}
\mathrm{N} & -2.536455 & -2.133552 & 0.763576
\end{array} \\
& \begin{array}{llll}
\text { C } & -3.562772 & -3.076954 & 0.739927
\end{array} \\
& \begin{array}{llll}
\text { C } & -3.197231 & -4.128779 & 1.552454
\end{array} \\
& \begin{array}{llll}
\mathrm{N} & -1.944523 & -3.810819 & 2.056450
\end{array} \\
& \begin{array}{llll}
\text { C } & -1.577748 & -2.607847 & 1.555466
\end{array} \\
& \begin{array}{lllll}
\text { C } & -4.796278 & -2.899364 & -0.106920
\end{array} \\
& \begin{array}{llll}
\text { C } & -5.787881 & -4.073436 & -0.050256
\end{array} \\
& \begin{array}{llll}
\mathrm{H} & 1.476141 & 1.225516 & -2.654927
\end{array} \\
& \mathrm{H} \quad 0.652498 \quad 0.106206 \quad-3.500221 \\
& \begin{array}{llll}
\mathrm{H} & 3.574747 & 0.353685 & -3.269739
\end{array} \\
& \text { H } \quad \begin{array}{llll}
1.916601 & -1.954213 & -4.419697
\end{array} \\
& \text { H } \quad 3.608235 \quad-1.698955-4.828912 \\
& \begin{array}{llll}
\mathrm{H} & 5.106385 & -3.526567 & -3.445319
\end{array} \\
& \begin{array}{llll}
\mathrm{H} & 3.032755 & -3.279866 & 0.250695
\end{array} \\
& \begin{array}{llll}
\mathrm{H} & 2.849217 & 6.190948 & -0.063870
\end{array} \\
& \begin{array}{llll}
\mathrm{H} & 1.143568 & 6.938971 & -1.732738
\end{array} \\
& \begin{array}{lllll}
\mathrm{H} & 1.972767 & 5.494364 & -2.315438
\end{array} \\
& \begin{array}{llll}
\mathrm{H} & -0.782550 & 5.472142 & -0.941771
\end{array} \\
& \text { H } \quad-0.520185 \quad 5.432078-2.682978 \\
& \begin{array}{llll}
\mathrm{H} & -5.324265 & -5.004832 & -0.394847
\end{array} \\
& \begin{array}{llll}
\mathrm{H} & -4.475491 & -2.741677 & -1.145980
\end{array} \\
& \begin{array}{llll}
\mathrm{H} & -5.303854 & -1.973606 & 0.192419
\end{array} \\
& \begin{array}{llll}
\mathrm{H} & -3.701584 & -5.050442 & 1.798796
\end{array} \\
& \begin{array}{llll}
\mathrm{H} & -0.628198 & -2.125751 & 1.758560
\end{array} \\
& \begin{array}{llll}
\mathrm{H} & -6.134230 & 1.667724 & 3.677267
\end{array} \\
& \begin{array}{llll}
\mathrm{H} & -7.721820 & 2.401721 & 1.135647
\end{array} \\
& \mathrm{H} \quad-6.609731 \quad 3.474390 \quad 1.977420 \\
& \text { H } \quad-5.525652 \quad 3.443216 \quad-0.567253 \\
& \begin{array}{llll}
\mathrm{H} & -4.897219 & -0.006826 & 1.756066
\end{array} \\
& \begin{array}{llll}
\mathrm{H} & -3.563645 & 2.181479 & -1.585058
\end{array} \\
& \begin{array}{llll}
\mathrm{H} & 7.692167 & 1.732139 & 2.439423
\end{array}
\end{aligned}
$$




$\begin{array}{cccc}\mathrm{H} & 6.040067 & 1.628903 & 5.038956 \\ \mathrm{H} & 5.861286 & 2.850767 & 3.786919 \\ \mathrm{H} & 5.855497 & -0.939974 & 4.046518 \\ \mathrm{H} & 4.007004 & 2.587795 & 2.362598 \\ \mathrm{H} & 4.091445 & -2.361585 & 3.026634 \\ \mathrm{H} & 2.222971 & 1.177475 & 1.342074 \\ \mathrm{H} & -0.995707 & 2.025116 & -1.293389 \\ \mathrm{H} & 8.244917 & 2.191340 & 4.062417 \\ \mathrm{H} & 7.866576 & 0.499813 & 3.701538 \\ \mathrm{H} & 2.638129 & 0.469922 & -4.764739 \\ \mathrm{H} & 1.227981 & 6.028714 & 0.631532 \\ \mathrm{H} & 2.087858 & 4.594636 & 0.031326 \\ \mathrm{H} & -6.163781 & -4.232551 & 0.967256 \\ \mathrm{H} & -6.647932 & -3.869808 & -0.695768 \\ \mathrm{H} & -7.860595 & 2.059566 & 3.597049 \\ \mathrm{H} & -7.259395 & 0.582657 & 2.831865 \\ \mathrm{H} & 4.981048 & -4.358899 & -1.022127 \\ \mathrm{H} & -1.390714 & -4.377060 & 2.685711 \\ \mathrm{O} & -1.150015 & 0.514894 & -1.467110 \\ \mathrm{O} & -0.357026 & -0.200828 & -0.489342\end{array}$


Table S13. Cartesian coordinates of Int 2 in the triplet state.
Atom $\quad \mathrm{x}$
$\mathrm{y}$
$\mathrm{Z}$
C $\quad 3.638223 \quad-2.188492 \quad 1.720779$
C $\quad 4.460555 \quad-2.944196 \quad 2.657138$
C $\quad 5.344447 \quad-2.302809 \quad 3.502416$
C $\quad 5.481812 \quad-0.884596 \quad 3.496904$
C $\quad 4.684634 \quad-0.127559 \quad 2.592303$
C $\quad 3.793115 \quad-0.739593 \quad 1.732156$
C $\quad 6.464387 \quad-0.205605 \quad 4.419501$
C $\quad 7.899473 \quad-0.150740 \quad 3.836635$
O $\quad 2.832038 \quad-2.766441 \quad 0.931676$
$\begin{array}{llll}\text { O } & -1.250963 & 3.234222 & 0.335668\end{array}$
C $\quad-0.235955 \quad 4.032860 \quad 0.021625$
O $\quad 0.550115 \quad 3.813775 \quad-0.899998$
$\begin{array}{llll}\text { C } & -0.165129 & 5.276639 & 0.897511\end{array}$
C $\quad 1.253239 \quad 5.844011 \quad 1.056080$
$\begin{array}{llll}\text { C } & 2.169691 & 4.947272 & 1.903131\end{array}$
$\begin{array}{llll}\mathrm{N} & 0.974756 & 1.893119 & -3.088778\end{array}$
$\begin{array}{llll}\text { C } & 2.216187 & 2.097293 & -3.865024\end{array}$
$\begin{array}{llll}\text { C } & 2.623667 & 0.826092 & -4.642945\end{array}$
$\begin{array}{llll}\text { C } & 3.161793 & -0.288928 & -3.788272\end{array}$
N $\quad 2.483494 \quad-0.795570 \quad-2.682378$
$\begin{array}{llll}\text { C } & 3.238230 & -1.759341 & -2.164961\end{array}$
$\begin{array}{llll}\mathrm{N} & 4.374612 & -1.902432 & -2.893276\end{array}$
C $\quad 4.346124 \quad-0.979885-3.926720$
$\begin{array}{llll}\mathrm{Cu} & 0.856323 & 0.040892 & -2.006150\end{array}$
$\begin{array}{llll}\mathrm{N} & -4.169574 & 0.490236 & 0.319068\end{array}$
$\begin{array}{llll}\text { C } & -4.902554 & 0.108756 & 1.433238\end{array}$
$\begin{array}{llll}\text { C } & -5.817419 & 1.088639 & 1.753916\end{array}$
$\begin{array}{llll}\mathrm{N} & -5.620670 & 2.073726 & 0.793857\end{array}$
$\begin{array}{llll}\text { C } & -4.623956 & 1.683265 & -0.040541\end{array}$
C $\quad-6.836473 \quad 1.209859 \quad 2.850859$ 


$$
\begin{aligned}
& \begin{array}{llll}
\text { C } & -6.692909 & 0.117767 & 3.921649
\end{array} \\
& \mathrm{Cu} \quad-2.478897 \quad-0.314768 \quad-0.389246 \\
& \begin{array}{llll}
\mathrm{N} & -2.415884 & -2.286307 & -0.063175
\end{array} \\
& \begin{array}{llll}
\text { C } & -3.356286 & -3.299522 & -0.242723
\end{array} \\
& \begin{array}{llll}
\text { C } & -2.762425 & -4.500541 & 0.082817
\end{array} \\
& \begin{array}{llll}
\mathrm{N} & -1.460498 & -4.200203 & 0.453607
\end{array} \\
& \begin{array}{llll}
\text { C } & -1.288631 & -2.862950 & 0.348366
\end{array} \\
& \begin{array}{llll}
\text { C } & -4.745333 & -3.028038 & -0.757309
\end{array} \\
& \begin{array}{llll}
\text { C } & -5.629151 & -4.278739 & -0.890576
\end{array} \\
& \begin{array}{llll}
\mathrm{H} & 0.849298 & 2.629323 & -2.381675
\end{array} \\
& \begin{array}{lllll}
\mathrm{H} & 0.163295 & 1.947138 & -3.704600
\end{array} \\
& \text { H } \quad 3.012979 \quad 2.369393 \quad-3.163775 \\
& \mathrm{H} \quad 1.752774 \quad 0.469537 \quad-5.212139 \\
& \begin{array}{llll}
\mathrm{H} & 3.390438 & 1.091518 & -5.379602
\end{array} \\
& \text { H } \quad 5.142776-0.894646 \quad-4.650755 \\
& \begin{array}{llll}
\text { H } & 3.010429 & -2.330176 & -1.272064
\end{array} \\
& \mathrm{H} \quad 3.166450 \quad 5.391988 \quad 2.005344 \\
& \mathrm{H} \quad 1.180780 \quad 6.835427 \quad 1.521086 \\
& \begin{array}{llll}
\mathrm{H} & 1.690360 & 5.985218 & 0.061319
\end{array} \\
& \begin{array}{llll}
\mathrm{H} & -0.618581 & 5.045314 & 1.867711
\end{array} \\
& \begin{array}{llll}
\mathrm{H} & -0.816441 & 6.020987 & 0.416728
\end{array} \\
& \begin{array}{llll}
\mathrm{H} & -5.195245 & -5.002953 & -1.589502
\end{array} \\
& \begin{array}{lllll}
\mathrm{H} & -4.658037 & -2.535110 & -1.735532
\end{array} \\
& \begin{array}{llll}
\mathrm{H} & -5.233428 & -2.299533 & -0.097897
\end{array} \\
& \begin{array}{llll}
\mathrm{H} & -3.142229 & -5.510545 & 0.073387
\end{array} \\
& \mathrm{H} \quad-0.355566 \quad-2.355990 \quad 0.558635 \\
& \begin{array}{llll}
\mathrm{H} & -5.707673 & 0.158776 & 4.398349
\end{array} \\
& \begin{array}{llll}
\mathrm{H} & -7.845530 & 1.170889 & 2.417074
\end{array} \\
& \begin{array}{llll}
\mathrm{H} & -6.738510 & 2.197992 & 3.321214
\end{array} \\
& \begin{array}{llll}
\mathrm{H} & -6.121963 & 2.951331 & 0.735016
\end{array} \\
& \begin{array}{llll}
\mathrm{H} & -4.712245 & -0.823546 & 1.944380
\end{array} \\
& \begin{array}{llll}
\mathrm{H} & -4.249505 & 2.279410 & -0.861024
\end{array} \\
& \begin{array}{llll}
\mathrm{H} & 7.921661 & 0.407464 & 2.894278
\end{array}
\end{aligned}
$$




$\begin{array}{cccc}\mathrm{H} & 6.494134 & -0.739602 & 5.377265 \\ \mathrm{H} & 6.126913 & 0.816172 & 4.628811 \\ \mathrm{H} & 5.947300 & -2.885304 & 4.197806 \\ \mathrm{H} & 4.778667 & 0.957345 & 2.590936 \\ \mathrm{H} & 4.346960 & -4.026007 & 2.661439 \\ \mathrm{H} & 3.173473 & -0.163693 & 1.047624 \\ \mathrm{H} & -1.303154 & 2.419114 & -0.289206 \\ \mathrm{H} & 8.571996 & 0.345074 & 4.544628 \\ \mathrm{H} & 8.287366 & -1.157101 & 3.644415 \\ \mathrm{H} & 2.116487 & 2.926645 & -4.580335 \\ \mathrm{H} & 1.761748 & 4.803140 & 2.911579 \\ \mathrm{H} & 2.288019 & 3.960735 & 1.439239 \\ \mathrm{H} & -5.768941 & -4.775840 & 0.076496 \\ \mathrm{H} & -6.617712 & -4.001749 & -1.269990 \\ \mathrm{H} & -7.451738 & 0.250353 & 4.698954 \\ \mathrm{H} & -6.828352 & -0.881072 & 3.491957 \\ \mathrm{H} & 5.111466 & -2.569220 & -2.705582 \\ \mathrm{H} & -0.748584 & -4.858483 & 0.743278 \\ \mathrm{O} & -1.579805 & 1.204500 & -1.198890 \\ \mathrm{O} & -0.626072 & 0.172592 & -0.822082\end{array}$


Table S14. Cartesian coordinates of Int 2 in the open-shell singlet state.
Atom $\quad \mathrm{x}$
$\mathrm{y}$
$\mathrm{Z}$
C $\quad 3.638536 \quad-2.189624 \quad 1.719495$
C $\quad 4.461105 \quad-2.945875 \quad 2.655245$
C $\quad 5.344889-2.304954 \quad 3.500978$
C $\quad 5.481996 \quad-0.886704 \quad 3.496485$
C $\quad 4.684670 \quad-0.129149 \quad 2.592431$
C $\quad 3.793222 \quad-0.740696 \quad 1.731879$
C $\quad 6.464488 \quad-0.208194 \quad 4.419525$
C $\quad 7.899578 \quad-0.152831 \quad 3.836719$
$\begin{array}{llll}\text { O } & 2.832315 & -2.767132 & 0.930137\end{array}$
$\begin{array}{llll}\text { O } & -1.251004 & 3.233777 & 0.337559\end{array}$
$\begin{array}{llll}\text { C } & -0.236235 & 4.032819 & 0.023769\end{array}$
O $\quad 0.549600 \quad 3.814528 \quad-0.898241$
$\begin{array}{llll}\text { C } & -0.165266 & 5.275915 & 0.900617\end{array}$
C $\quad 1.253095 \quad 5.843290 \quad 1.059279$
C $\quad 2.169811 \quad 4.946084 \quad 1.905554$
$\begin{array}{llll}\mathrm{N} & 0.974711 & 1.895284 & -3.087831\end{array}$
$\begin{array}{llll}\text { C } & 2.216139 & 2.099838 & -3.863941\end{array}$
$\begin{array}{llll}\text { C } & 2.623704 & 0.829025 & -4.642468\end{array}$
$\begin{array}{llll}\text { C } & 3.161845 & -0.286461 & -3.788424\end{array}$
N $\quad 2.483429 \quad-0.793894 \quad-2.682946$
$\begin{array}{llll}\text { C } & 3.238248 & -1.757920 & -2.166069\end{array}$
N $4.374729 \quad-1.900432 \quad-2.894339$
C $\quad 4.346256 \quad-0.977216 \quad-3.927185$
$\begin{array}{llll}\mathrm{Cu} & 0.856204 & 0.042204 & -2.006405\end{array}$
$\begin{array}{llll}\mathrm{N} & -4.169629 & 0.489962 & 0.319293\end{array}$
$\begin{array}{llll}\text { C } & -4.902527 & 0.107928 & 1.433331\end{array}$
$\begin{array}{llll}\text { C } & -5.817481 & 1.087585 & 1.754461\end{array}$
$\begin{array}{llll}\mathrm{N} & -5.620857 & 2.073107 & 0.794837\end{array}$
$\begin{array}{llll}\text { C } & -4.624149 & 1.683103 & -0.039780\end{array}$
$\begin{array}{llll}\text { C } & -6.836538 & 1.208174 & 2.851471\end{array}$ 


$$
\begin{aligned}
& \text { C } \quad-6.692803 \quad 0.115656 \quad 3.921795 \\
& \begin{array}{lllll}
\mathrm{Cu} & -2.479017 & -0.314644 & -0.389467
\end{array} \\
& \begin{array}{llll}
\mathrm{N} & -2.415891 & -2.286331 & -0.064214
\end{array} \\
& \begin{array}{llll}
\text { C } & -3.356210 & -3.299549 & -0.244221
\end{array} \\
& \begin{array}{llll}
\text { C } & -2.762266 & -4.500660 & 0.080826
\end{array} \\
& \begin{array}{llll}
\mathrm{N} & -1.460373 & -4.200388 & 0.451771
\end{array} \\
& \begin{array}{llll}
\text { C } & -1.288592 & -2.863071 & 0.347109
\end{array} \\
& \begin{array}{llll}
\text { C } & -4.745275 & -3.027931 & -0.758707
\end{array} \\
& \begin{array}{llll}
\text { C } & -5.629031 & -4.278621 & -0.892463
\end{array} \\
& \begin{array}{llll}
\mathrm{H} & 0.849308 & 2.630987 & -2.380215
\end{array} \\
& \mathrm{H} \quad 0.163228 \quad 1.949655 \quad-3.703581 \\
& \text { H } \quad 3.012916 \quad 2.371613 \quad-3.162554 \\
& \mathrm{H} \quad 1.752854 \quad 0.472743 \quad-5.211903 \\
& \begin{array}{llll}
\mathrm{H} & 3.390492 & 1.094878 & -5.378959
\end{array} \\
& \begin{array}{llll}
\mathrm{H} & 5.142985 & -0.891426 & -4.651070
\end{array} \\
& \begin{array}{llll}
\mathrm{H} & 3.010326 & -2.329324 & -1.273578
\end{array} \\
& \begin{array}{llll}
\mathrm{H} & 3.166579 & 5.390780 & 2.007756
\end{array} \\
& \mathrm{H} \quad 1.180649 \quad 6.834393 \quad 1.524957 \\
& \begin{array}{llll}
\mathrm{H} & 1.690004 & 5.985200 & 0.064525
\end{array} \\
& \begin{array}{llll}
\mathrm{H} & -0.618473 & 5.043827 & 1.870750
\end{array} \\
& \begin{array}{llll}
\mathrm{H} & -0.816737 & 6.020593 & 0.420558
\end{array} \\
& \begin{array}{llll}
\mathrm{H} & -5.195094 & -5.002542 & -1.591675
\end{array} \\
& \text { H } \quad-4.657985-2.534608 \quad-1.736732 \\
& \begin{array}{llll}
\mathrm{H} & -5.233394 & -2.299703 & -0.099004
\end{array} \\
& \begin{array}{llll}
\mathrm{H} & -3.141996 & -5.510687 & 0.070935
\end{array} \\
& \mathrm{H} \quad-0.355564 \quad-2.356152 \quad 0.557643 \\
& \begin{array}{llll}
\mathrm{H} & -5.707563 & 0.156594 & 4.398495
\end{array} \\
& \begin{array}{llll}
\mathrm{H} & -7.845592 & 1.169220 & 2.417672
\end{array} \\
& \begin{array}{llll}
\mathrm{H} & -6.738745 & 2.196125 & 3.322244
\end{array} \\
& \begin{array}{llll}
\mathrm{H} & -6.122282 & 2.950660 & 0.736337
\end{array} \\
& \begin{array}{llll}
\mathrm{H} & -4.712104 & -0.824575 & 1.944063
\end{array} \\
& \text { H } \quad-4.249758 \quad 2.279659 \quad-0.859993 \\
& \begin{array}{llll}
\mathrm{H} & 7.921741 & 0.405937 & 2.894697
\end{array}
\end{aligned}
$$



H $\quad 6.494266 \quad-0.742792 \quad 5.376954$
H $\quad 6.126900 \quad 0.813415 \quad 4.629470$
H $\quad 5.947866 \quad-2.887847 \quad 4.195925$
H $4.778566 \quad 0.955768 \quad 2.591809$
H $\quad 4.347683 \quad-4.027708 \quad 2.658745$
H $3.173524 \quad-0.164401 \quad 1.047738$
H $\quad-1.303320 \quad 2.419154 \quad-0.287950$
H $\quad 8.572041 \quad 0.342623 \quad 4.545019$
H $\quad 8.287579 \quad-1.159039 \quad 3.643908$
H $2.116456 \quad 2.929523 \quad-4.578876$
H $\quad 1.762147 \quad 4.801337 \quad 2.914026$
H $\quad 2.288036 \quad 3.959831 \quad 1.441034$
H $\quad-5.768791 \quad-4.776115 \quad 0.074413$
H $\quad-6.617616 \quad-4.001557 \quad-1.271763$
H $\quad-7.451632 \quad 0.247831 \quad 4.699170$
H $\quad-6.828123 \quad-0.883023 \quad 3.491695$
H $\quad 5.111641 \quad-2.567250 \quad-2.706989$
H $\quad-0.748417 \quad-4.858750 \quad 0.741150$
$\begin{array}{llll}\text { O } & -1.580061 & 1.205138 & -1.198370\end{array}$
$\begin{array}{llll}\text { O } & -0.626242 & 0.173062 & -0.822186\end{array}$ 
Table S15. Cartesian coordinates of TS2 in the triplet state.
Atom $\mathrm{x}$
$\mathrm{y}$
$\mathrm{Z}$
C $\quad 3.520579 \quad-2.821638 \quad 0.717300$
$\begin{array}{llll}\text { C } & 4.394399 & -3.929644 & 1.080967\end{array}$
C $\quad 5.349643 \quad-3.785801 \quad 2.068570$
C $\quad 5.512744 \quad-2.557309 \quad 2.770662$
C $\quad 4.666234 \quad-1.463023 \quad 2.432629$
C $\quad 3.703077 \quad-1.574184 \quad 1.448393$
C $\quad 6.571131 \quad-2.414184 \quad 3.836934$
C $\quad 7.934432 \quad-1.939586 \quad 3.272101$
O $\quad 2.649276-2.938165 \quad-0.195744$
$\begin{array}{llll}\text { O } & -1.010219 & 2.518718 & 1.454407\end{array}$
C $\quad-0.168905 \quad 3.543072 \quad 1.477609$
O $\quad 0.446323 \quad 3.954258 \quad 0.491842$
$\begin{array}{llll}\text { C } & -0.060519 & 4.197924 & 2.848267\end{array}$
C $\quad 1.325587 \quad 4.792830 \quad 3.141493$
C $\quad 2.411982 \quad 3.722335 \quad 3.329926$
$\mathrm{N} \quad 1.025939 \quad 3.414525 \quad-2.281161$
$\begin{array}{llll}\text { C } & 2.416150 & 3.734727 & -2.697663\end{array}$
$\begin{array}{llll}\text { C } & 2.925088 & 2.799401 & -3.813864\end{array}$
C $\quad 3.249550 \quad 1.401531 \quad-3.359094$
N $\quad 2.370562 \quad 0.635377 \quad-2.602723$
$\begin{array}{llll}\text { C } & 2.960525 & -0.532199 & -2.380188\end{array}$
N $4.185353 \quad-0.556298 \quad-2.966948$
$\begin{array}{llll}\text { C } & 4.389600 & 0.662555 & -3.593201\end{array}$
$\begin{array}{llll}\mathrm{Cu} & 0.636454 & 1.445460 & -1.914373\end{array}$
$\begin{array}{llll}\mathrm{N} & -4.049488 & 0.341181 & 0.561806\end{array}$
$\begin{array}{llll}\text { C } & -4.789474 & -0.368617 & 1.495314\end{array}$
$\begin{array}{llll}\text { C } & -5.659591 & 0.476317 & 2.150898\end{array}$
$\begin{array}{llll}\mathrm{N} & -5.428345 & 1.721936 & 1.580271\end{array}$
$\begin{array}{llll}\text { C } & -4.456297 & 1.600356 & 0.639135\end{array}$
$\begin{array}{llll}\text { C } & -6.666257 & 0.255806 & 3.243813\end{array}$ 


$$
\begin{aligned}
& \begin{array}{llll}
\text { C } & -6.630523 & -1.171254 & 3.810617
\end{array} \\
& \mathrm{Cu} \quad-2.480147 \quad-0.266986 \quad-0.524560 \\
& \begin{array}{llll}
\mathrm{N} & -2.472233 & -2.229728 & -0.958824
\end{array} \\
& \begin{array}{llll}
\text { C } & -3.465729 & -3.088744 & -1.422497
\end{array} \\
& \begin{array}{llll}
\text { C } & -2.913427 & -4.341513 & -1.587682
\end{array} \\
& \begin{array}{llll}
\mathrm{N} & -1.580881 & -4.225849 & -1.221926
\end{array} \\
& \begin{array}{llll}
\text { C } & -1.353799 & -2.942451 & -0.855079
\end{array} \\
& \begin{array}{llll}
\text { C } & -4.866759 & -2.614320 & -1.703684
\end{array} \\
& \begin{array}{llll}
\text { C } & -5.812683 & -3.703649 & -2.233903
\end{array} \\
& \begin{array}{llll}
\mathrm{H} & 0.816215 & 3.832755 & -1.364638
\end{array} \\
& \begin{array}{llll}
\mathrm{H} & 0.366461 & 3.809818 & -2.951589
\end{array} \\
& \text { H } \quad 3.057799 \quad 3.641151 \quad-1.815181 \\
& \begin{array}{llll}
\mathrm{H} & 2.174423 & 2.766539 & -4.617176
\end{array} \\
& \begin{array}{llll}
\mathrm{H} & 3.828782 & 3.237121 & -4.252807
\end{array} \\
& \begin{array}{llll}
\mathrm{H} & 5.295191 & 0.892992 & -4.134773
\end{array} \\
& \text { H } 2.563173 \quad-1.353184 \quad-1.793976 \\
& \mathrm{H} \quad 3.381901 \quad 4.183048 \quad 3.550155 \\
& \mathrm{H} \quad 1.255411 \quad 5.405523 \quad 4.049118 \\
& \mathrm{H} \quad 1.603727 \quad 5.463238 \quad 2.320690 \\
& \text { H } \quad-0.354925 \quad 3.467057 \quad 3.609094 \\
& \begin{array}{llll}
\mathrm{H} & -0.819075 & 4.993951 & 2.863276
\end{array} \\
& \begin{array}{llll}
\mathrm{H} & -5.448187 & -4.123739 & -3.178337
\end{array} \\
& \text { H } \quad-4.810084 \quad-1.791644 \quad-2.429832 \\
& \begin{array}{llll}
\mathrm{H} & -5.283236 & -2.176245 & -0.787490
\end{array} \\
& \begin{array}{llll}
\mathrm{H} & -3.339567 & -5.271567 & -1.931141
\end{array} \\
& \mathrm{H} \quad-0.389130 \quad-2.567583 \quad-0.537137 \\
& \begin{array}{llll}
\mathrm{H} & -5.647758 & -1.404006 & 4.234776
\end{array} \\
& \begin{array}{llll}
\mathrm{H} & -7.672984 & 0.474289 & 2.860568
\end{array} \\
& \begin{array}{llll}
\mathrm{H} & -6.479437 & 0.975274 & 4.053208
\end{array} \\
& \begin{array}{llll}
\mathrm{H} & -5.895554 & 2.583784 & 1.832173
\end{array} \\
& \begin{array}{llll}
\mathrm{H} & -4.638058 & -1.426694 & 1.651687
\end{array} \\
& \begin{array}{llll}
\mathrm{H} & -4.068571 & 2.424129 & 0.056172
\end{array} \\
& \text { H } \quad 7.842972 \quad-0.960139 \quad 2.789885
\end{aligned}
$$




$$
\begin{array}{crrr}
\mathrm{H} & 6.714323 & -3.376720 & 4.342355 \\
\mathrm{H} & 6.233357 & -1.697320 & 4.594789 \\
\mathrm{H} & 5.989738 & -4.627600 & 2.328947 \\
\mathrm{H} & 4.780409 & -0.524803 & 2.973640 \\
\mathrm{H} & 4.260024 & -4.867820 & 0.547079 \\
\mathrm{H} & 3.045318 & -0.744747 & 1.196425 \\
\mathrm{H} & -1.115003 & 2.101893 & 0.513003 \\
\mathrm{H} & 8.666025 & -1.855234 & 4.082641 \\
\mathrm{H} & 8.323695 & -2.647503 & 2.532169 \\
\mathrm{H} & 2.486720 & 4.774375 & -3.045490 \\
\mathrm{H} & 2.163662 & 3.048769 & 4.159770 \\
\mathrm{H} & 2.527069 & 3.116988 & 2.422492 \\
\mathrm{H} & -5.924167 & -4.523025 & -1.514008 \\
\mathrm{H} & -6.806367 & -3.283105 & -2.417949 \\
\mathrm{H} & -7.375902 & -1.280364 & 4.604446 \\
\mathrm{H} & -6.856965 & -1.912267 & 3.035847 \\
\mathrm{H} & 4.830315 & -1.335157 & -2.944465 \\
\mathrm{H} & -0.887375 & -4.962329 & -1.232488 \\
\mathrm{O} & -1.490010 & 1.436961 & -0.815040 \\
\mathrm{O} & -0.620894 & 0.278715 & -1.061335
\end{array}
$$


Table S16. Cartesian coordinates of TS2 in the open-shell singlet state.
Atom $\mathrm{x} \quad \mathrm{y} \quad \mathrm{z}$
C $\quad 3.520581 \quad-2.821601 \quad 0.716822$
C $\quad 4.393773 \quad-3.930078 \quad 1.080548$
$\begin{array}{llll}\text { C } & 5.348988 & -3.786772 & 2.068274\end{array}$
C $\quad 5.512669 \quad-2.558398 \quad 2.770414$
C $\quad 4.666785 \quad-1.463637 \quad 2.432300$
C $\quad 3.703693 \quad-1.574255 \quad 1.447951$
C $\quad 6.570964 \quad-2.415827 \quad 3.836861$
C $\quad 7.934061 \quad-1.939967 \quad 3.272595$
$\begin{array}{llll}\text { O } & 2.649287 & -2.937632 & -0.196298\end{array}$
O $\quad-1.010320 \quad 2.518440 \quad 1.454840$
C $\quad-0.169008 \quad 3.542797 \quad 1.478292$
O $\quad 0.446401 \quad 3.954066 \quad 0.492677$
C $\quad-0.060883 \quad 4.197538 \quad 2.849023$
C $\quad 1.325181 \quad 4.792394 \quad 3.142550$
C $\quad 2.411515 \quad 3.721854 \quad 3.331089$
$\mathrm{N} \quad 1.025824 \quad 3.415123 \quad-2.280501$
C $\quad 2.415954 \quad 3.735436 \quad-2.697180$
C $\quad 2.924672 \quad 2.800395 \quad-3.813720$
$\begin{array}{llll}\text { C } & 3.249316 & 1.402440 & -3.359348\end{array}$
N $\quad 2.370515 \quad 0.636034 \quad-2.603011$
$\begin{array}{llll}\text { C } & 2.960622 & -0.531540 & -2.380853\end{array}$
N $4.185359 \quad-0.555395 \quad-2.967822$
$\begin{array}{llll}\text { C } & 4.389392 & 0.663623 & -3.593823\end{array}$
$\begin{array}{llll}\mathrm{Cu} & 0.636449 & 1.445951 & -1.914222\end{array}$
$\begin{array}{llll}\mathrm{N} & -4.049151 & 0.340982 & 0.562044\end{array}$
$\begin{array}{llll}\text { C } & -4.789578 & -0.369014 & 1.495053\end{array}$
$\begin{array}{llll}\text { C } & -5.659320 & 0.475958 & 2.151085\end{array}$
$\begin{array}{llll}\mathrm{N} & -5.427386 & 1.721811 & 1.581247\end{array}$
$\begin{array}{llll}\text { C } & -4.455323 & 1.600317 & 0.640119\end{array}$
$\begin{array}{llll}\text { C } & -6.666207 & 0.255305 & 3.243769\end{array}$ 


$$
\begin{aligned}
& \text { C } \quad-6.630952-1.171975 \quad 3.810045 \\
& \mathrm{Cu} \quad-2.479907 \quad-0.267078 \quad-0.524573 \\
& \begin{array}{llll}
\mathrm{N} & -2.472135 & -2.229763 & -0.959094
\end{array} \\
& \begin{array}{llll}
\text { C } & -3.465639 & -3.088577 & -1.423138
\end{array} \\
& \begin{array}{llll}
\text { C } & -2.913458 & -4.341390 & -1.588384
\end{array} \\
& \begin{array}{llll}
\mathrm{N} & -1.580982 & -4.225951 & -1.222299
\end{array} \\
& \begin{array}{llll}
\text { C } & -1.353825 & -2.942641 & -0.855197
\end{array} \\
& \begin{array}{llll}
\text { C } & -4.866541 & -2.613936 & -1.704615
\end{array} \\
& \begin{array}{llll}
\text { C } & -5.812565 & -3.703150 & -2.234912
\end{array} \\
& \begin{array}{lllll}
\mathrm{H} & 0.816257 & 3.833100 & -1.363829
\end{array} \\
& \mathrm{H} \quad 0.366217 \quad 3.810586 \quad-2.950700 \\
& \text { H } 3.057764 \quad 3.641623 \quad-1.814840 \\
& \text { H } \quad 2.173808 \quad 2.767686 \quad-4.616851 \\
& \begin{array}{llll}
\mathrm{H} & 3.828237 & 3.238260 & -4.252783
\end{array} \\
& \begin{array}{llll}
\mathrm{H} & 5.294870 & 0.894263 & -4.135498
\end{array} \\
& \text { H } \quad 2.563440 \quad-1.352690 \quad-1.794759 \\
& \mathrm{H} \quad 3.381412 \quad 4.182522 \quad 3.551502 \\
& \mathrm{H} \quad 1.254853 \quad 5.405020 \quad 4.050209 \\
& \text { H } 1.603479 \quad 5.462856 \quad 2.321846 \\
& \begin{array}{llll}
\mathrm{H} & -0.355445 & 3.466616 & 3.609737
\end{array} \\
& \begin{array}{llll}
\mathrm{H} & -0.819425 & 4.993581 & 2.863948
\end{array} \\
& \begin{array}{llll}
\mathrm{H} & -5.447992 & -4.123364 & -3.179261
\end{array} \\
& \text { H } \quad-4.809599 \quad-1.791333 \quad-2.430823 \\
& \text { H } \quad-5.283106 \quad-2.175713 \quad-0.788531 \\
& \text { H } \quad-3.339640 \quad-5.271339-1.932073 \\
& \begin{array}{llll}
\mathrm{H} & -0.389163 & -2.567955 & -0.536994
\end{array} \\
& \begin{array}{llll}
\mathrm{H} & -5.648326 & -1.405145 & 4.234297
\end{array} \\
& \begin{array}{llll}
\mathrm{H} & -7.672819 & 0.474204 & 2.860457
\end{array} \\
& \begin{array}{llll}
\mathrm{H} & -6.479315 & 0.974420 & 4.053460
\end{array} \\
& \begin{array}{llll}
\mathrm{H} & -5.894176 & 2.583743 & 1.833640
\end{array} \\
& \begin{array}{llll}
\mathrm{H} & -4.638709 & -1.427262 & 1.650789
\end{array} \\
& \begin{array}{llll}
\mathrm{H} & -4.067123 & 2.424253 & 0.057705
\end{array} \\
& \text { H } \quad 7.842144 \quad-0.960080 \quad 2.791361
\end{aligned}
$$




$\begin{array}{lrrr}\mathrm{H} & 6.714596 & -3.378779 & 4.341353 \\ \mathrm{H} & 6.232790 & -1.699873 & 4.595406 \\ \mathrm{H} & 5.988607 & -4.628915 & 2.328701 \\ \mathrm{H} & 4.781394 & -0.525489 & 2.973345 \\ \mathrm{H} & 4.258962 & -4.868170 & 0.546624 \\ \mathrm{H} & 3.046429 & -0.744451 & 1.195902 \\ \mathrm{H} & -1.114925 & 2.101703 & 0.513375 \\ \mathrm{H} & 8.665553 & -1.856060 & 4.083272 \\ \mathrm{H} & 8.323723 & -2.646943 & 2.531976 \\ \mathrm{H} & 2.486464 & 4.775175 & -3.044747 \\ \mathrm{H} & 2.163042 & 3.048242 & 4.160849 \\ \mathrm{H} & 2.526727 & 3.116559 & 2.423634 \\ \mathrm{H} & -5.924291 & -4.522455 & -1.514974 \\ \mathrm{H} & -6.806150 & -3.282453 & -2.419145 \\ \mathrm{H} & -7.376510 & -1.281190 & 4.603691 \\ \mathrm{H} & -6.857444 & -1.912638 & 3.034955 \\ \mathrm{H} & 4.830396 & -1.334199 & -2.945627 \\ \mathrm{H} & -0.887562 & -4.962514 & -1.232825 \\ \mathrm{O} & -1.489912 & 1.436984 & -0.814782 \\ \mathrm{O} & -0.620787 & 0.278845 & -1.061487\end{array}$


Table S17. Cartesian coordinates of Int 3 in the triplet state.
Atom $\quad x$
$\mathrm{y}$
$\mathrm{Z}$
$\begin{array}{llll}\mathrm{N} & 0.407923 & 2.865027 & -3.197617\end{array}$
C $\quad 1.809462 \quad 2.958279 \quad-3.683270$
H $\quad 1.912208 \quad 3.784057 \quad-4.399118$
C $\quad 2.293888 \quad 1.651420 \quad-4.342795$
C $\quad 2.471276 \quad 0.524995 \quad-3.364364$
$\mathrm{N} \quad 1.448428 \quad 0.112193 \quad-2.521631$
C $\quad 3.585519 \quad-0.243751 \quad-3.098102$
C $\quad 1.941939 \quad-0.874074 \quad-1.770380$
N $\quad 3.229230 \quad-1.119847 \quad-2.091163$
H $\quad 2.427566 \quad 3.828361 \quad 1.026539$
C $\quad 2.380010 \quad 4.567440 \quad 1.835085$
H $\quad 2.286991 \quad 4.027896 \quad 2.786286$
$\begin{array}{llll}\text { C } & 1.202513 & 5.533124 & 1.627484\end{array}$
$\begin{array}{llll}\text { C } & -0.164545 & 4.833220 & 1.613355\end{array}$
$\begin{array}{llll}\text { C } & -0.408453 & 3.964457 & 0.386786\end{array}$
$\begin{array}{llll}\text { O } & -1.304615 & 3.012675 & 0.589636\end{array}$
$\begin{array}{llll}\text { O } & 0.160301 & 4.159933 & -0.692017\end{array}$
$\begin{array}{llll}\mathrm{H} & -5.768278 & -5.213751 & 1.599559\end{array}$
$\begin{array}{llll}\text { C } & -5.204259 & -5.190592 & 0.661643\end{array}$
$\begin{array}{llll}\mathrm{H} & -4.527292 & -6.052851 & 0.661371\end{array}$
$\begin{array}{llll}\text { C } & -4.432567 & -3.869807 & 0.536939\end{array}$
$\begin{array}{llll}\text { C } & -3.627994 & -3.737449 & -0.726143\end{array}$
$\begin{array}{llll}\mathrm{N} & -2.986273 & -2.548268 & -1.059096\end{array}$
$\begin{array}{llll}\text { C } & -3.384539 & -4.659143 & -1.721355\end{array}$
$\begin{array}{llll}\text { C } & -2.375698 & -2.745534 & -2.222206\end{array}$
$\begin{array}{llll}\mathrm{N} & -2.589200 & -4.012165 & -2.655970\end{array}$
$\begin{array}{llll}\mathrm{H} & -6.649875 & -1.036841 & 4.333229\end{array}$
$\begin{array}{llll}\text { C } & -6.089979 & -0.352198 & 4.979762\end{array}$
$\begin{array}{llll}\mathrm{H} & -6.651127 & -0.236007 & 5.912182\end{array}$
$\begin{array}{llll}\text { C } & -5.895538 & 1.014499 & 4.304372\end{array}$ 

$\begin{array}{llll}C & -5.129963 & 0.919740 & 3.016109\end{array}$
$\begin{array}{llll}\mathrm{N} & -4.853344 & 2.016914 & 2.209434\end{array}$
$\begin{array}{llll}\text { C } & -4.562684 & -0.144827 & 2.352541\end{array}$
$\begin{array}{llll}\text { C } & -4.149613 & 1.600045 & 1.123798\end{array}$
$\begin{array}{llll}\mathrm{N} & -3.956814 & 0.289286 & 1.180894\end{array}$
H $\quad 9.262436 \quad-0.401189 \quad 3.266634$
$\begin{array}{llll}\text { C } & 8.471873 & -0.214243 & 4.001135\end{array}$
$\mathrm{H} \quad 8.944445 \quad 0.023638 \quad 4.959929$
$\begin{array}{llll}\text { C } & 7.552152 & -1.450081 & 4.157303\end{array}$
$\begin{array}{llll}\text { C } & 6.857620 & -1.812426 & 2.870059\end{array}$
$\begin{array}{llll}\text { C } & 5.791460 & -0.999852 & 2.381664\end{array}$
$\begin{array}{llll}\text { C } & 7.260185 & -2.942056 & 2.105238\end{array}$
$\begin{array}{llll}\text { C } & 5.157533 & -1.285360 & 1.191923\end{array}$
$\begin{array}{llll}\text { C } & 6.642279 & -3.257141 & 0.910047\end{array}$
$\begin{array}{llll}\text { C } & 5.559073 & -2.435129 & 0.394177\end{array}$
$\begin{array}{llll}\text { O } & 4.996400 & -2.721061 & -0.707476\end{array}$
$\mathrm{Cu} \quad-0.203243 \quad 1.156327 \quad-2.245402$
$\mathrm{Cu} \quad-3.071615 \quad-0.825846 \quad-0.134085$
$\begin{array}{llll}\mathrm{H} & 1.198915 & 6.280705 & 2.430838\end{array}$
$\begin{array}{llll}\mathrm{H} & 1.331819 & 6.075565 & 0.684351\end{array}$
$\begin{array}{llll}\mathrm{H} & -0.314060 & 4.216677 & 2.507048\end{array}$
$\begin{array}{llll}\mathrm{H} & -0.969909 & 5.582221 & 1.615362\end{array}$
$\mathrm{H} \quad 3.332277 \quad 5.109912 \quad 1.849605$
$\begin{array}{llll}\mathrm{H} & 6.799327 & -1.231847 & 4.926345\end{array}$
H $\quad 8.143414 \quad-2.299875 \quad 4.515026$
$\begin{array}{llll}\mathrm{H} & 8.070291 & -3.567205 & 2.476531\end{array}$
$\begin{array}{llll}\mathrm{H} & 5.475154 & -0.139519 & 2.969477\end{array}$
$\begin{array}{llll}\mathrm{H} & 6.939640 & -4.118954 & 0.317395\end{array}$
H $4.344253 \quad-0.664949 \quad 0.821505$
$\begin{array}{llll}\mathrm{H} & -1.491316 & 2.432825 & -0.264384\end{array}$
$\begin{array}{llll}\mathrm{H} & 7.906122 & 0.664908 & 3.674018\end{array}$
H $\quad 0.250590 \quad 3.543806 \quad-2.437059$
H $2.442602 \quad 3.190859 \quad-2.820973$ 


$\begin{array}{lrrr}\mathrm{H} & 3.252799 & 1.842073 & -4.836976 \\ \mathrm{H} & 1.585100 & 1.362448 & -5.132265 \\ \mathrm{H} & 4.574317 & -0.237216 & -3.532749 \\ \mathrm{H} & 1.398220 & -1.402759 & -0.999171 \\ \mathrm{H} & 3.863537 & -1.781810 & -1.598236 \\ \mathrm{H} & -5.917119 & -5.313742 & -0.161557 \\ \mathrm{H} & -3.754919 & -3.753251 & 1.394733 \\ \mathrm{H} & -5.132321 & -3.024556 & 0.581529 \\ \mathrm{H} & -3.699750 & -5.684165 & -1.842053 \\ \mathrm{H} & -1.802882 & -1.999647 & -2.755746 \\ \mathrm{H} & -2.243113 & -4.406235 & -3.520645 \\ \mathrm{H} & -5.126974 & -0.814865 & 5.222410 \\ \mathrm{H} & -6.876784 & 1.470136 & 4.110650 \\ \mathrm{H} & -5.369192 & 1.691502 & 4.991920 \\ \mathrm{H} & -5.117394 & 2.974991 & 2.399117 \\ \mathrm{H} & -4.551399 & -1.183667 & 2.645296 \\ \mathrm{H} & -3.795287 & 2.253299 & 0.339900 \\ \mathrm{H} & -0.242186 & 3.086412 & -3.952151 \\ \mathrm{O} & -1.897279 & 1.615698 & -1.448154 \\ \mathrm{O} & -1.576723 & 0.207173 & -1.234411\end{array}$


Table S18. Cartesian coordinates of Int3 in the open-shell singlet state.
Atom $\mathrm{x} \quad \mathrm{y} \quad \mathrm{z}$
$\mathrm{N} \quad-0.601781 \quad-2.179847 \quad-3.648526$
C $\quad-2.005611 \quad-2.086879-4.126557$
H $\quad-2.162951-2.753582 \quad-4.984300$
C $\quad-2.394825 \quad-0.648760 \quad-4.521532$
C $\quad-2.508887 \quad 0.282505 \quad-3.347433$
$\mathrm{N} \quad-1.489204 \quad 0.431082 \quad-2.416482$
C $\quad-3.558667 \quad 1.093467 \quad-2.968315$
C $\quad-1.922267 \quad 1.305227 \quad-1.506242$
N $\quad-3.165798 \quad 1.731852-1.807982$
H $\quad-2.056563 \quad-3.987333 \quad 0.966693$
C $\quad-1.983568-4.980820 \quad 1.426755$
H $\quad-1.528163 \quad-4.862140 \quad 2.417990$
$\begin{array}{llll}\text { C } & -1.162166 & -5.938402 & 0.548601\end{array}$
$\begin{array}{llll}\text { C } & 0.284763 & -5.466622 & 0.326835\end{array}$
C $\quad 0.405225 \quad-4.253106 \quad-0.586028$
$\begin{array}{llll}\text { O } & 1.349491 & -3.402297 & -0.226863\end{array}$
$\begin{array}{llll}\text { O } & -0.304634 & -4.112538 & -1.588182\end{array}$
H $\quad 5.977447 \quad 4.961067 \quad 1.812326$
$\begin{array}{llll}\text { C } & 5.424354 & 4.965420 & 0.867629\end{array}$
H $4.718206 \quad 5.8032890 .898306$
$\begin{array}{llll}\text { C } & 4.697308 & 3.627814 & 0.670588\end{array}$
$\begin{array}{llll}\text { C } & 3.901936 & 3.533621 & -0.602086\end{array}$
N $\quad 3.161707 \quad 2.397654 \quad-0.918974$
$\begin{array}{llll}\text { C } & 3.756596 & 4.448017 & -1.622617\end{array}$
$\begin{array}{llll}\text { C } & 2.589156 & 2.620060 & -2.096814\end{array}$
$\begin{array}{llll}\mathrm{N} & 2.923460 & 3.850343 & -2.556983\end{array}$
H $\quad 7.072583 \quad 0.143970 \quad 4.024330$
C $\quad 6.414366 \quad-0.128610 \quad 4.856541$
H $\quad 7.040878 \quad-0.306409 \quad 5.736142$
$\begin{array}{llll}\text { C } & 5.594306 & -1.384197 & 4.525491\end{array}$ 


$$
\begin{array}{lrrr}
\mathrm{C} & 4.712841 & -1.192202 & 3.325834 \\
\mathrm{~N} & 3.887375 & -2.186179 & 2.816447 \\
\mathrm{C} & 4.502852 & -0.114980 & 2.495085 \\
\mathrm{C} & 3.225326 & -1.703039 & 1.731568 \\
\mathrm{~N} & 3.580161 & -0.442617 & 1.510076 \\
\mathrm{H} & -8.937615 & -0.187912 & 3.214988 \\
\mathrm{C} & -8.147334 & -0.459304 & 3.922894 \\
\mathrm{H} & -8.615983 & -0.916297 & 4.800864 \\
\mathrm{C} & -7.328668 & 0.784948 & 4.346644 \\
\mathrm{C} & -6.650885 & 1.441344 & 3.172137 \\
\mathrm{C} & -5.525386 & 0.814647 & 2.559214 \\
\mathrm{C} & -7.130570 & 2.665083 & 2.629175 \\
\mathrm{C} & -4.913087 & 1.362697 & 1.453667 \\
\mathrm{C} & -6.534546 & 3.243330 & 1.524456 \\
\mathrm{C} & -5.398066 & 2.608354 & 0.876516 \\
\mathrm{H} & -4.862773 & 3.127291 & -0.151383 \\
\mathrm{H} & -0.472513 & -3.028504 & -3.073556 \\
\mathrm{H} & 0.071399 & -0.771694 & -2.327068 \\
\mathrm{Cu} & 3.019487 & 0.728963 & 0.073956 \\
\mathrm{H} & -1.134990 & -6.929607 & 1.018349 \\
\mathrm{H} & -1.645536 & -6.058640 & -0.427269 \\
\mathrm{H} & 0.782445 & -5.245906 & 1.277344 \\
\mathrm{H} & 0.863578 & -6.264098 & -0.160440 \\
\mathrm{H} & -3.002695 & -5.358108 & 1.569367 \\
\mathrm{H} & -6.570712 & 0.474655 & 5.077991 \\
\mathrm{H} & -7.988488 & 1.501365 & 4.847789 \\
\mathrm{H} & -7.984575 & 3.149925 & 3.098733 \\
\mathrm{H} & -2.434994 & -3.316430
\end{array}
$$



H $\quad-3.358347 \quad-0.674927 \quad-5.042057$
H $\quad-1.659415 \quad-0.264330 \quad-5.243442$
H $\quad-4.525797 \quad 1.261534 \quad-3.419015$
H $\quad-1.364552 \quad 1.629355 \quad-0.637933$
H $\quad-3.759048 \quad 2.341765 \quad-1.207241$
H $\quad 6.143918 \quad 5.151727 \quad 0.061985$
Н $\quad 4.017938 \quad 3.444754 \quad 1.514649$
H $\quad 5.426365 \quad 2.805500 \quad 0.678685$
H $\quad 4.161972 \quad 5.438538 \quad-1.760557$
H $\quad 1.951415 \quad 1.920914 \quad-2.619138$
H $\quad 2.622362 \quad 4.254728 \quad-3.433700$
H $\quad 5.761795 \quad 0.723796 \quad 5.075511$
H $\quad 6.273379 \quad-2.229612 \quad 4.346465$
H $\quad 4.976756 \quad-1.659352 \quad 5.392154$
H $\quad 3.796390 \quad-3.126064 \quad 3.179843$
H $\quad 4.955979 \quad 0.863321 \quad 2.544614$
H $\quad 2.524665 \quad-2.286987 \quad 1.149478$
H $\quad 0.035492 \quad-2.247609 \quad-4.442676$
O $\quad 1.773945 \quad-1.452708 \quad-1.721750$
$\begin{array}{llll}\text { O } & 1.378382 & -0.261032 & -0.979471\end{array}$ 
Table S19. Cartesian coordinates of TS3 in the triplet state.
Atom $\mathrm{x} \quad \mathrm{y} \quad \mathrm{z}$
$\mathrm{N} \quad-0.085587 \quad-3.309112 \quad-2.218729$
C $\quad-1.379619-3.934042 \quad-2.596059$
H $\quad-1.219029-4.949870 \quad-2.981638$
C $\quad-2.144335-3.108790 \quad-3.648115$
C $\quad-2.675991-1.806337 \quad-3.120023$
N $\quad-1.872879 \quad-0.904614 \quad-2.439048$
C $\quad-3.951573-1.282868-3.191978$
C $\quad-2.646175 \quad 0.129382 \quad-2.114325$
$\mathrm{N} \quad-3.908825 \quad-0.057951 \quad-2.554283$
$\mathrm{H} \quad-1.870321 \quad-3.689659 \quad 2.297191$
C $\quad-1.649743 \quad-4.227863 \quad 3.227177$
H $\quad-1.642718 \quad-3.499453 \quad 4.047769$
C $\quad-0.306554-4.968797 \quad 3.131986$
C $\quad 0.887200 \quad-4.030004 \quad 2.894746$
$\begin{array}{llll}\text { C } & 0.909533 & -3.404723 & 1.507674\end{array}$
O $\quad 1.441610 \quad-2.190089 \quad 1.487402$
$\begin{array}{llll}\text { O } & 0.499860 & -3.991550 & 0.502874\end{array}$
H $\quad 6.479575 \quad 4.125252 \quad-2.404238$
C $\quad 5.540338 \quad 4.464486 \quad-1.955767$
H $\quad 5.791312 \quad 5.111444 \quad-1.106835$
C $\quad 4.696633 \quad 3.260292 \quad-1.514288$
$\begin{array}{llll}\text { C } & 3.381243 & 3.637298 & -0.890471\end{array}$
$\begin{array}{llll}\mathrm{N} & 2.466378 & 2.685739 & -0.452414\end{array}$
$\begin{array}{llll}\text { C } & 2.843101 & 4.890630 & -0.682647\end{array}$
$\begin{array}{llll}\text { C } & 1.406936 & 3.345439 & -0.000018\end{array}$
$\begin{array}{llll}\mathrm{N} & 1.594620 & 4.683018 & -0.117093\end{array}$
H $\quad 7.120151 \quad 2.125341 \quad 2.892668$
$\begin{array}{llll}\text { C } & 7.060568 & 1.237483 & 3.532038\end{array}$
H $\quad 7.890797 \quad 1.282450 \quad 4.243598$
$\begin{array}{llll}\text { C } & 7.143908 & -0.052794 & 2.702869\end{array}$ 

$\begin{array}{llll}\text { C } & 6.026992 & -0.183532 & 1.706636\end{array}$
$\begin{array}{llll}\mathrm{N} & 5.824793 & -1.332781 & 0.952155\end{array}$
C $\quad 5.014919 \quad 0.667206 \quad 1.313869$
$\begin{array}{llll}C & 4.733355 & -1.151814 & 0.162586\end{array}$
$\begin{array}{llll}\mathrm{N} & 4.220661 & 0.053743 & 0.358198\end{array}$
$\begin{array}{llll}\mathrm{H} & -8.406802 & 0.701272 & 4.071772\end{array}$
$\begin{array}{llll}\text { C } & -7.501752 & 1.136833 & 4.508243\end{array}$
$\mathrm{H} \quad-7.697570 \quad 1.343656 \quad 5.565705$
$\begin{array}{llll}\text { C } & -7.104711 & 2.441629 & 3.778575\end{array}$
$\begin{array}{llll}\text { C } & -6.807694 & 2.224348 & 2.316473\end{array}$
$\begin{array}{llll}\text { C } & -5.677333 & 1.445835 & 1.927058\end{array}$
$\begin{array}{llll}\text { C } & -7.642214 & 2.769108 & 1.302712\end{array}$
$\begin{array}{llll}\text { C } & -5.387149 & 1.214989 & 0.600296\end{array}$
$\begin{array}{llll}\text { C } & -7.375818 & 2.559445 & -0.037700\end{array}$
$\begin{array}{llll}\text { C } & -6.227587 & 1.769046 & -0.452521\end{array}$
$\begin{array}{llll}\text { O } & -5.977182 & 1.581772 & -1.682213\end{array}$
$\mathrm{Cu} \quad-0.045948 \quad-1.295923 \quad-1.739826$
$\begin{array}{llll}\mathrm{Cu} & 2.453764 & 0.635739 & -0.439569\end{array}$
$\mathrm{H} \quad-0.129762 \quad-5.523807 \quad 4.061856$
$\begin{array}{llll}\mathrm{H} & -0.343937 & -5.703978 & 2.320410\end{array}$
$\begin{array}{llll}\mathrm{H} & 0.926191 & -3.228226 & 3.639853\end{array}$
H $\quad \begin{array}{llll}1.827626 & -4.592678 & 2.986215\end{array}$
H $\quad-2.471543 \quad-4.929777 \quad 3.409019$
$\mathrm{H} \quad-6.213194 \quad 2.858884 \quad 4.267366$
$\begin{array}{llll}\mathrm{H} & -7.905423 & 3.180581 & 3.889819\end{array}$
$\begin{array}{llll}\mathrm{H} & -8.505731 & 3.363677 & 1.595498\end{array}$
$\begin{array}{llll}\mathrm{H} & -5.034583 & 1.028145 & 2.700318\end{array}$
$\begin{array}{llll}\mathrm{H} & -8.005455 & 2.973073 & -0.821842\end{array}$
$\begin{array}{llll}\mathrm{H} & -4.529596 & 0.613401 & 0.307638\end{array}$
$\begin{array}{llll}\mathrm{H} & 1.470470 & -1.792837 & 0.537431\end{array}$
$\mathrm{H} \quad-6.705076 \quad 0.387163 \quad 4.453423$
H $\quad 0.252037 \quad-3.716909-1.334161$
H $\quad-1.980884 \quad-4.020606 \quad-1.685043$ 

Н $\quad-2.983491 \quad-3.706018 \quad-4.021423$
H $\quad-1.483906 \quad-2.926031 \quad-4.508596$
H $\quad-4.857028 \quad-1.671827 \quad-3.634246$

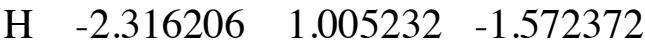
H $\quad-4.705767 \quad 0.570839 \quad-2.340381$
H $\quad 5.0141115 .068468 \quad-2.703907$
H $\quad 5.264677 \quad 2.645752 \quad-0.804407$
H $\quad 4.494553 \quad 2.612706 \quad-2.378471$
H $\quad 3.224830 \quad 5.878252 \quad-0.891129$
$\mathrm{H} \quad 0.509670 \quad 2.889879 \quad 0.397050$
H $\quad 0.935478 \quad 5.400138 \quad 0.155314$
H $\quad 6.124623 \quad 1.282851 \quad 4.099369$
H $\quad 8.108014 \quad-0.089651 \quad 2.176227$
H $\quad 7.125462 \quad-0.920760 \quad 3.376699$
H $\quad 6.390574 \quad-2.171192 \quad 0.989135$
H $\quad 4.799907 \quad 1.663490 \quad 1.672044$
H $\quad 4.338130 \quad-1.899655 \quad-0.511503$
H $\quad 0.613849 \quad-3.503251 \quad-2.936153$
$\begin{array}{llll}\text { O } & 1.653322 & -1.116515 & -0.848029\end{array}$
$\begin{array}{llll}\text { O } & 0.608180 & 0.290382 & -0.893814\end{array}$ 
Table S20. Cartesian coordinates of TS3 in the open-shell singlet state.
Atom $\mathrm{x} \quad \mathrm{y} \quad \mathrm{z}$
$\begin{array}{llll}\mathrm{N} & 0.085230 & 3.309741 & -2.217923\end{array}$
$\begin{array}{llll}\text { C } & 1.379245 & 3.934887 & -2.594946\end{array}$
H $\quad 1.218612 \quad 4.950830-2.980203$
$\begin{array}{llll}\text { C } & 2.144098 & 3.110034 & -3.647213\end{array}$
C $\quad 2.675806 \quad 1.807447 \quad-3.119503$
$\begin{array}{llll}\mathrm{N} & 1.872697 & 0.905410 & -2.438942\end{array}$
$\begin{array}{llll}\text { C } & 3.951449 & 1.284129 & -3.191486\end{array}$
$\begin{array}{llll}\text { C } & 2.646057 & -0.128622 & -2.114484\end{array}$
$\begin{array}{llll}\mathrm{N} & 3.908745 & 0.058989 & -2.554221\end{array}$
H $\quad 1.870681 \quad 3.688983 \quad 2.297251$
$\begin{array}{llll}\text { C } & 1.650360 & 4.226791 & 3.227527\end{array}$
H $\quad 1.643690 \quad 3.498051 \quad 4.047829$
$\begin{array}{llll}\text { C } & 0.307059 & 4.967619 & 3.133092\end{array}$
$\begin{array}{llll}\text { C } & -0.886678 & 4.028788 & 2.895900\end{array}$
$\begin{array}{llll}\text { C } & -0.909392 & 3.404032 & 1.508598\end{array}$
$\begin{array}{llll}\text { O } & -1.441272 & 2.189315 & 1.488038\end{array}$
$\begin{array}{llll}\text { O } & -0.500161 & 3.991313 & 0.503884\end{array}$
H $\quad-6.480007-4.125202-2.404230$
$\begin{array}{llll}\text { C } & -5.540657 & -4.464437 & -1.955996\end{array}$
H $\quad-5.791416 \quad-5.111487-1.107072$
$\begin{array}{llll}\text { C } & -4.696907 & -3.260248 & -1.514583\end{array}$
$\begin{array}{llll}\text { C } & -3.381377 & -3.637258 & -0.891057\end{array}$
$\begin{array}{llll}\mathrm{N} & -2.466477 & -2.685703 & -0.453062\end{array}$
$\begin{array}{llll}\text { C } & -2.843129 & -4.890587 & -0.683489\end{array}$
$\begin{array}{llll}\text { C } & -1.406913 & -3.345402 & -0.000954\end{array}$
$\begin{array}{llll}\mathrm{N} & -1.594549 & -4.682978 & -0.118153\end{array}$
$\mathrm{H} \quad-7.120124 \quad-2.125571 \quad 2.892686$
$\begin{array}{llll}\text { C } & -7.060449 & -1.237771 & 3.532127\end{array}$
H $\quad-7.890610 \quad-1.282772 \quad 4.243765$
$\begin{array}{llll}\text { C } & -7.143828 & 0.052581 & 2.703079\end{array}$ 


$$
\begin{array}{lrrr}
\mathrm{C} & -6.027006 & 0.183380 & 1.706749 \\
\mathrm{~N} & -5.824855 & 1.332696 & 0.952356 \\
\mathrm{C} & -5.014990 & -0.667343 & 1.313801 \\
\mathrm{C} & -4.733499 & 1.151777 & 0.162662 \\
\mathrm{~N} & -4.220812 & -0.053811 & 0.358109 \\
\mathrm{H} & 8.406849 & -0.702536 & 4.071741 \\
\mathrm{C} & 7.501906 & -1.138558 & 4.507977 \\
\mathrm{H} & 7.697735 & -1.345788 & 5.565358 \\
\mathrm{C} & 7.105259 & -2.443151 & 3.777720 \\
\mathrm{C} & 6.808147 & -2.225221 & 2.315738 \\
\mathrm{C} & 5.677044 & -1.447557 & 1.926776 \\
\mathrm{C} & 7.643313 & -2.768426 & 1.301676 \\
\mathrm{C} & 5.386745 & -1.216068 & 0.600151 \\
\mathrm{C} & 7.376825 & -2.558084 & -0.038611 \\
\mathrm{C} & 6.227830 & -1.768551 & -0.452978 \\
\mathrm{H} & -0.252495 & 3.717240 & -1.333257 \\
\mathrm{O} & 5.977334 & -1.580686 & -1.682558 \\
\mathrm{Cu} & 0.045707 & 1.296394 & -1.739650 \\
\mathrm{Cu} & -2.453968 & -0.635705 & -0.439886 \\
\mathrm{H} & 0.130531 & 5.522243 & 4.063242 \\
\mathrm{H} & 0.344077 & 5.703121 & 2.321791 \\
\mathrm{H} & -0.925337 & 3.226729 & 3.640721 \\
\mathrm{H} & -1.827131 & 4.591332 & 2.987886 \\
\mathrm{H} & 2.472148 & 4.928719 & 3.409371 \\
\mathrm{H} & 6.213873 & -2.860890 & 4.266329 \\
\mathrm{H} & 7.906201 & -3.181907 & 3.888604 \\
\mathrm{H} & 5.0307383 & -3.362359 & 1.594122 \\
\mathrm{H} & -1.031053 & 2.700289 \\
\mathrm{H} & -1.470388 & 1.792448 & 0.537923 \\
\mathrm{H} & -2.970542 & -0.822991 \\
\mathrm{H} & -0.615007 & -0.389105 & 4.453432 \\
\mathrm{H} & -1.683856
\end{array}
$$



$\begin{array}{llll}\mathrm{H} & 2.983239 & 3.707441 & -4.020264\end{array}$
H $\quad 1.483745 \quad 2.927505 \quad-4.507802$
$\begin{array}{lllll}\mathrm{H} & 4.856918 & 1.673331 & -3.633510\end{array}$
$\mathrm{H} \quad 2.316104 \quad-1.004696 \quad-1.572882$
H $\quad 4.705718 \quad-0.569792 \quad-2.340455$
$\begin{array}{llll}\mathrm{H} & -5.014567 & -5.068324 & -2.704309\end{array}$
$\mathrm{H} \quad-5.264832 \quad-2.645787 \quad-0.804539$
$\mathrm{H} \quad-4.495021 \quad-2.612585 \quad-2.378753$
$\mathrm{H} \quad-3.224847 \quad-5.878204 \quad-0.892016$
$\begin{array}{lllll}\mathrm{H} & -0.509595 & -2.889843 & 0.395997\end{array}$
H $\quad-0.935316 \quad-5.400096 \quad 0.154038$
$\begin{array}{lllll}\mathrm{H} & -6.124450 & -1.283221 & 4.099363\end{array}$
$\begin{array}{lllll}\mathrm{H} & -8.107984 & 0.089513 & 2.176533\end{array}$
$\begin{array}{lllll}\mathrm{H} & -7.125294 & 0.920487 & 3.376986\end{array}$
$\begin{array}{llll}\mathrm{H} & -6.390618 & 2.171114 & 0.989467\end{array}$

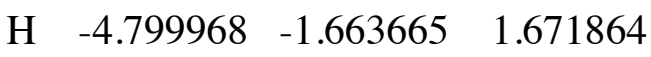

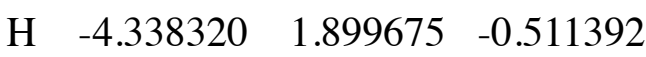
$\begin{array}{lllll}\mathrm{H} & -0.614157 & 3.504044 & -2.935350\end{array}$
$\begin{array}{lllll}\mathrm{O} & -1.653485 & 1.116676 & -0.847815\end{array}$
$\begin{array}{lllll}\text { O } & -0.608417 & -0.290229 & -0.894270\end{array}$ 
Table S21. Cartesian coordinates of BO in the triplet state.
Atom
$\mathrm{x}$
$\mathrm{y}$
$\mathrm{Z}$
$\mathrm{N} \quad-0.039368 \quad 2.533872 \quad-2.558970$
C $\quad 1.203615 \quad 3.046013 \quad-3.187299$
H $\quad 0.973172 \quad 3.945346 \quad-3.772793$
C $\quad 1.888206 \quad 2.006630 \quad-4.088462$
C $\quad 2.375194 \quad 0.819749 \quad-3.311660$
N $\quad 1.572616 \quad 0.182738-2.380415$
C $\quad 3.584759 \quad 0.158925 \quad-3.346193$
C $\quad 2.270216 \quad-0.832617 \quad-1.873421$
N $\quad 3.492724 \quad-0.878996 \quad-2.436286$
H $\quad 1.777643 \quad 4.465741 \quad 1.585006$
C $\quad 1.462228 \quad 5.198124 \quad 2.337355$
H $\quad 1.443073 \quad 4.698570 \quad 3.314360$
C $\quad 0.085566 \quad 5.786092 \quad 1.987453$
$\begin{array}{llll}\text { C } & -1.034068 & 4.736623 & 1.925848\end{array}$
$\begin{array}{llll}\text { C } & -0.974769 & 3.803544 & 0.722602\end{array}$
$\begin{array}{llll}\text { O } & -1.670767 & 2.701187 & 0.870567\end{array}$
$\begin{array}{llll}\text { O } & -0.345054 & 4.095264 & -0.308526\end{array}$
H $\quad-6.006779 \quad-4.363024 \quad-2.071147$
$\begin{array}{llll}\text { C } & -5.174970 & -4.604301 & -1.401763\end{array}$
H $\quad-5.595798 \quad-5.071041 \quad-0.503324$
$\begin{array}{llll}\text { C } & -4.385349 & -3.332512 & -1.057137\end{array}$
C $\quad-3.216008-3.572906-0.141885$
$\mathrm{N} \quad-2.343233 \quad-2.558406 \quad 0.240378$
$\begin{array}{llll}\text { C } & -2.779777 & -4.754550 & 0.420647\end{array}$
C $\quad-1.405705 \quad-3.112836 \quad 0.999045$
N $\quad-1.636419 \quad-4.440551 \quad 1.138783$
H $\quad-6.722657-1.834381 \quad 3.242251$
C $\quad-6.741256-0.851928 \quad 3.727224$
H $\quad-7.572247 \quad-0.847191 \quad 4.439304$
$\begin{array}{llll}\text { C } & -6.920152 & 0.274083 & 2.697762\end{array}$ 


$$
\begin{aligned}
& \begin{array}{llll}
\text { C } & -5.809234 & 0.324858 & 1.687632
\end{array} \\
& \begin{array}{llll}
\mathrm{N} & -5.674309 & 1.356259 & 0.765206
\end{array} \\
& \begin{array}{llll}
\text { C } & -4.747303 & -0.513089 & 1.423763
\end{array} \\
& \begin{array}{llll}
\text { C } & -4.572182 & 1.127230 & 0.006416
\end{array} \\
& \begin{array}{llll}
\mathrm{N} & -3.989822 & -0.002801 & 0.381439
\end{array} \\
& \text { H } \quad 8.574459 \quad 0.797246 \quad 3.256303 \\
& \begin{array}{llll}
\text { C } & 7.788783 & 0.507490 & 3.962033
\end{array} \\
& \text { H } \quad 8.136853 \quad 0.737050 \quad 4.974679 \\
& \begin{array}{llll}
\text { C } & 7.463493 & -1.000985 & 3.843473
\end{array} \\
& \begin{array}{llll}
\text { C } & 6.964169 & -1.374493 & 2.471398
\end{array} \\
& \begin{array}{llll}
\text { C } & 5.688561 & -0.912837 & 2.029684
\end{array} \\
& \begin{array}{llll}
\text { C } & 7.748223 & -2.162800 & 1.585256
\end{array} \\
& \begin{array}{llll}
\text { C } & 5.216877 & -1.204313 & 0.768945
\end{array} \\
& \begin{array}{llll}
\text { C } & 7.300227 & -2.478226 & 0.315937
\end{array} \\
& \begin{array}{llll}
\text { C } & 6.009453 & -2.002652 & -0.156164
\end{array} \\
& \begin{array}{llll}
\text { O } & 5.603836 & -2.276532 & -1.327482
\end{array} \\
& \mathrm{Cu} \quad-0.153710 \quad 0.728071 \quad-1.723668 \\
& \mathrm{Cu} \quad-2.197616 \quad-0.599231 \quad-0.319816 \\
& \begin{array}{llll}
\mathrm{H} & -0.188516 & 6.537552 & 2.739121
\end{array} \\
& \begin{array}{llll}
\mathrm{H} & 0.140401 & 6.302730 & 1.022672
\end{array} \\
& \begin{array}{llll}
\mathrm{H} & -1.061257 & 4.119356 & 2.831589
\end{array} \\
& \begin{array}{llll}
\mathrm{H} & -2.013953 & 5.232716 & 1.868424
\end{array} \\
& \begin{array}{llll}
\mathrm{H} & 2.224197 & 5.984840 & 2.379183
\end{array} \\
& \begin{array}{llll}
\mathrm{H} & 6.696252 & -1.253625 & 4.588084
\end{array} \\
& \begin{array}{llll}
\mathrm{H} & 8.355565 & -1.587031 & 4.089320
\end{array} \\
& \begin{array}{llll}
\mathrm{H} & 8.719872 & -2.521526 & 1.919947
\end{array} \\
& \begin{array}{llll}
\mathrm{H} & 5.081055 & -0.316912 & 2.708957
\end{array} \\
& \begin{array}{llll}
\mathrm{H} & 7.890932 & -3.080206 & -0.370422
\end{array} \\
& \text { H } \quad 4.246640 \quad-0.844350 \quad 0.434930 \\
& \begin{array}{llll}
\mathrm{H} & -1.658985 & 2.047770 & -0.006209
\end{array} \\
& \mathrm{H} \quad 6.906922 \quad 1.124868 \quad 3.759599 \\
& \text { H } \quad-0.288715 \quad 3.151308 \quad-1.753082 \\
& \begin{array}{llll}
\text { H } & 1.879135 & 3.343939 & -2.379143
\end{array}
\end{aligned}
$$



H $\quad 2.734870 \quad 2.478222 \quad-4.598104$
H $\quad 1.187023 \quad 1.684783 \quad-4.872424$
H $\quad 4.473167 \quad 0.335833 \quad-3.934325$
H $\quad 1.884414 \quad-1.511859-1.126867$
H $\quad 4.264237 \quad-1.499444 \quad-2.119157$
Н $\quad-4.542729 \quad-5.342271 \quad-1.908640$
H $\quad-5.052292 \quad-2.592289 \quad-0.597059$
H $\quad-4.008930 \quad-2.869451 \quad-1.979555$
H $\quad-3.167382 \quad-5.759964 \quad 0.363256$
H $\quad-0.561335 \quad-2.593610 \quad 1.430424$
$\begin{array}{llll}\mathrm{H} & -1.066007 & -5.087281 & 1.667311\end{array}$
H $\quad-5.810360 \quad-0.726991 \quad 4.290826$
H $\quad-7.880496 \quad 0.148034 \quad 2.178112$
H $\quad-6.974298 \quad 1.239788 \quad 3.219378$
H $\quad-6.284298 \quad 2.159643 \quad 0.682438$
H $\quad-4.475931 \quad-1.431391 \quad 1.922926$
H $\quad-4.208532 \quad 1.783446 \quad-0.771547$
H $\quad-0.819270 \quad 2.561815 \quad-3.217593$
$\begin{array}{llll}\text { O } & -1.779415 & 1.159101 & -1.055472\end{array}$
$\begin{array}{llll}\text { O } & -0.414693 & -0.841248 & -0.905071\end{array}$ 
Table S22. Cartesian coordinates of BO in the open-shell singlet state.
Atom $\mathrm{x} \quad \mathrm{y} \quad \mathrm{z}$
$\mathrm{N} \quad 0.039714 \quad-2.534160 \quad-2.559292$
C $\quad-1.203359-3.046312-3.187482$
Н $\quad-0.972956 \quad-3.945613-3.773038$
C $\quad-1.888119-2.006949-4.088527$
C $\quad-2.375007-0.820024 \quad-3.311733$
$\mathrm{N} \quad-1.572271-0.182904-2.380675$
C $\quad-3.584483-0.159056-3.346325$
C $\quad-2.269736 \quad 0.832596 \quad-1.873805$
N $\quad-3.492260 \quad 0.879047 \quad-2.436629$
Н $\quad-1.778354-4.465971 \quad 1.585000$
C $\quad-1.462720 \quad-5.198044 \quad 2.337564$
H $\quad-1.444107 \quad-4.698303 \quad 3.314483$
C $\quad-0.085633 \quad-5.785280 \quad 1.988140$
C $\quad 1.033394 \quad-4.735186 \quad 1.926546$
$\begin{array}{llll}\text { C } & 0.974192 & -3.802631 & 0.722879\end{array}$
$\begin{array}{llll}\text { O } & 1.670693 & -2.700466 & 0.870342\end{array}$
$\begin{array}{llll}\text { O } & 0.344280 & -4.094479 & -0.308056\end{array}$
H $\quad 6.006411 \quad 4.362858 \quad-2.071445$
$\begin{array}{llll}\text { C } & 5.174762 & 4.604182 & -1.401879\end{array}$
H $\quad 5.595811 \quad 5.070449 \quad-0.503304$
C $\quad 4.384753 \quad 3.332517 \quad-1.057639$
C $3.215635 \quad 3.572953-0.142117$
$\begin{array}{llll}\mathrm{N} & 2.343296 & 2.558367 & 0.240956\end{array}$
$\begin{array}{llll}\text { C } & 2.779281 & 4.754726 & 0.420041\end{array}$
C $\quad 1.405923 \quad 3.112884 \quad 0.999743$
N $\quad 1.636307 \quad 4.440728 \quad 1.138785$
H $\quad 6.722651 \quad 1.834230 \quad 3.242188$
$\begin{array}{llll}\text { C } & 6.740765 & 0.851921 & 3.727473\end{array}$
H $\quad 7.571506 \quad 0.847166 \quad 4.439850$
$\begin{array}{llll}\text { C } & 6.919645 & -0.274472 & 2.698434\end{array}$ 


$$
\begin{aligned}
& \begin{array}{llll}
C & 5.809015 & -0.325172 & 1.687988
\end{array} \\
& \begin{array}{llll}
\mathrm{N} & 5.674016 & -1.356740 & 0.765752
\end{array} \\
& \begin{array}{llll}
\text { C } & 4.747233 & 0.512875 & 1.423850
\end{array} \\
& \begin{array}{llll}
\text { C } & 4.572022 & -1.127688 & 0.006786
\end{array} \\
& \begin{array}{llll}
\mathrm{N} & 3.989731 & 0.002441 & 0.381611
\end{array} \\
& \begin{array}{llll}
\mathrm{H} & -8.574919 & -0.796763 & 3.256308
\end{array} \\
& \begin{array}{llll}
\text { C } & -7.789139 & -0.507223 & 3.962012
\end{array} \\
& \mathrm{H} \quad-8.137231 \quad-0.736716 \quad 4.974667 \\
& \begin{array}{llll}
\text { C } & -7.463454 & 1.001165 & 3.843461
\end{array} \\
& \begin{array}{llll}
\text { C } & -6.964083 & 1.374535 & 2.471364
\end{array} \\
& \begin{array}{llll}
\text { C } & -5.688548 & 0.912667 & 2.029665
\end{array} \\
& \begin{array}{llll}
\text { C } & -7.748043 & 2.162901 & 1.585190
\end{array} \\
& \begin{array}{llll}
\text { C } & -5.216847 & 1.204010 & 0.768901
\end{array} \\
& \begin{array}{llll}
\text { C } & -7.300025 & 2.478203 & 0.315847
\end{array} \\
& \begin{array}{llll}
\text { C } & -6.009317 & 2.002432 & -0.156232
\end{array} \\
& \begin{array}{llll}
\text { O } & -5.603664 & 2.276150 & -1.327568
\end{array} \\
& \mathrm{Cu} \quad 0.154159 \quad-0.728296 \quad-1.724164 \\
& \begin{array}{lllll}
\mathrm{Cu} & 2.197735 & 0.599190 & -0.319560
\end{array} \\
& \begin{array}{llll}
\mathrm{H} & 0.188713 & -6.536389 & 2.740063
\end{array} \\
& \begin{array}{llll}
\mathrm{H} & -0.139932 & -6.302212 & 1.023479
\end{array} \\
& \begin{array}{llll}
\mathrm{H} & 1.059810 & -4.117462 & 2.831998
\end{array} \\
& \begin{array}{llll}
\mathrm{H} & 2.013614 & -5.230699 & 1.869813
\end{array} \\
& \begin{array}{llll}
\mathrm{H} & -2.224264 & -5.985177 & 2.379328
\end{array} \\
& \begin{array}{llll}
\mathrm{H} & -6.696122 & 1.253598 & 4.588048
\end{array} \\
& \begin{array}{llll}
\mathrm{H} & -8.355364 & 1.587444 & 4.089342
\end{array} \\
& \begin{array}{llll}
\mathrm{H} & -8.719636 & 2.521779 & 1.919882
\end{array} \\
& \begin{array}{llll}
\mathrm{H} & -5.081130 & 0.316683 & 2.708964
\end{array} \\
& \begin{array}{llll}
\mathrm{H} & -7.890660 & 3.080227 & -0.370533
\end{array} \\
& \begin{array}{llll}
\mathrm{H} & -4.246671 & 0.843903 & 0.434852
\end{array} \\
& \begin{array}{llll}
\mathrm{H} & 1.659161 & -2.047148 & -0.006795
\end{array} \\
& \text { H } \quad-6.907450 \quad-1.124831 \quad 3.759530 \\
& \begin{array}{lllll}
\mathrm{H} & 0.288995 & -3.151489 & -1.753324
\end{array} \\
& \text { H } \quad-1.878742 \quad-3.344288 \quad-2.379231
\end{aligned}
$$




$\begin{array}{lrrr}\mathrm{H} & -2.734848 & -2.478573 & -4.598031 \\ \mathrm{H} & -1.187071 & -1.685092 & -4.872606 \\ \mathrm{H} & -4.472915 & -0.335883 & -3.934447 \\ \mathrm{H} & -1.883769 & 1.511900 & -1.127391 \\ \mathrm{H} & -4.263777 & 1.499430 & -2.119436 \\ \mathrm{H} & 4.542716 & 5.342531 & -1.908448 \\ \mathrm{H} & 5.051495 & 2.591880 & -0.597935 \\ \mathrm{H} & 4.008070 & 2.869959 & -1.980206 \\ \mathrm{H} & 3.166604 & 5.760214 & 0.362073 \\ \mathrm{H} & 0.561904 & 2.593638 & 1.431791 \\ \mathrm{H} & 1.066011 & 5.087504 & 1.667383 \\ \mathrm{H} & 5.809643 & 0.727460 & 4.290806 \\ \mathrm{H} & 7.880217 & -0.148945 & 2.179080 \\ \mathrm{H} & 6.973265 & -1.240025 & 3.220388 \\ \mathrm{H} & 6.283861 & -2.160258 & 0.683240 \\ \mathrm{H} & 4.475915 & 1.431299 & 1.922815 \\ \mathrm{H} & 4.208428 & -1.783926 & -0.771178 \\ \mathrm{H} & 0.819596 & -2.562272 & -3.217929 \\ \mathrm{O} & 1.779999 & -1.159144 & -1.056056 \\ \mathrm{O} & 0.415223 & 0.841104 & -0.905727\end{array}$


Table S23. Cartesian coordinates of $\mathrm{OHO}$ in the triplet state.

$\begin{array}{cccc}\text { Atom } & \mathrm{x} & \mathrm{y} & \mathrm{z} \\ \mathrm{N} & 0.099141 & 2.501706 & -2.532876 \\ \mathrm{C} & 1.359647 & 2.942768 & -3.176733 \\ \mathrm{H} & 1.162756 & 3.846207 & -3.767544 \\ \mathrm{C} & 1.992833 & 1.868287 & -4.074755 \\ \mathrm{C} & 2.417595 & 0.670055 & -3.281411 \\ \mathrm{~N} & 1.573570 & 0.075018 & -2.358340 \\ \mathrm{C} & 3.600844 & -0.036431 & -3.276903 \\ \mathrm{C} & 2.220779 & -0.958423 & -1.818785 \\ \mathrm{~N} & 3.451075 & -1.056010 & -2.353828 \\ \mathrm{H} & 1.687964 & 4.410796 & 1.790961 \\ \mathrm{C} & 1.329566 & 5.239346 & 2.412822 \\ \mathrm{H} & 1.087643 & 4.842205 & 3.407247 \\ \mathrm{C} & 0.103902 & 5.908922 & 1.769715 \\ \mathrm{C} & -1.094269 & 4.964820 & 1.587512 \\ \mathrm{C} & -0.960425 & 3.917662 & 0.476484 \\ \mathrm{O} & -1.813799 & 2.961203 & 0.501354 \\ \mathrm{O} & -0.065159 & 4.057187 & -0.405266 \\ \mathrm{H} & -5.903677 & -4.398795 & -2.235412 \\ \mathrm{C} & -5.118074 & -4.619297 & -1.506000 \\ \mathrm{H} & -5.598719 & -5.044626 & -0.617207 \\ \mathrm{C} & -4.337979 & -3.339002 & -1.165386 \\ \mathrm{C} & -3.233270 & -3.549330 & -0.166239 \\ \mathrm{~N} & -2.391813 & -2.521509 & 0.251193 \\ \mathrm{C} & -2.835350 & -4.712810 & 0.458835 \\ \mathrm{C} & -1.510358 & -3.051233 & 1.091201 \\ \mathrm{H} & -1.747814 & -4.375182 & 1.248593 \\ \mathrm{H} & -6.813636 & -1.916513 & 3.093864 \\ \mathrm{H} & -6.731156 & -1.018226 & 3.716117 \\ \mathrm{H} & -1.040998 & 4.443805 \\ \mathrm{H} & 0.257756 & 2.866184\end{array}$




$$
\begin{array}{cccc}
\mathrm{C} & -5.716563 & 0.355795 & 1.846669 \\
\mathrm{~N} & -5.502638 & 1.495617 & 1.080269 \\
\mathrm{C} & -4.736836 & -0.521586 & 1.437837 \\
\mathrm{C} & -4.437387 & 1.290928 & 0.269022 \\
\mathrm{~N} & -3.949941 & 0.071018 & 0.459424 \\
\mathrm{H} & 8.545832 & 0.892274 & 3.160126 \\
\mathrm{C} & 7.767149 & 0.652725 & 3.891930 \\
\mathrm{H} & 8.128219 & 0.945165 & 4.883557 \\
\mathrm{C} & 7.434080 & -0.858746 & 3.875351 \\
\mathrm{C} & 6.916643 & -1.317581 & 2.536593 \\
\mathrm{C} & 5.643051 & -0.868595 & 2.075750 \\
\mathrm{C} & 7.680785 & -2.175819 & 1.699165 \\
\mathrm{C} & 5.155829 & -1.237701 & 0.841786 \\
\mathrm{C} & 7.216305 & -2.569941 & 0.458125 \\
\mathrm{C} & 5.928427 & -2.107709 & -0.034087 \\
\mathrm{O} & 5.508758 & -2.453069 & -1.181903 \\
\mathrm{Cu} & -0.135423 & 0.689169 & -1.781746 \\
\mathrm{Cu} & -2.236309 & -0.581640 & -0.351046 \\
\mathrm{H} & -0.212348 & 6.755100 & 2.394695 \\
\mathrm{H} & 0.382551 & 6.317779 & 0.792043 \\
\mathrm{H} & -1.330352 & 4.433137 & 2.517700 \\
\mathrm{H} & -1.994139 & 5.545525 & 1.337709 \\
\mathrm{H} & 2.152417 & 5.954479 & 2.529622 \\
\mathrm{H} & 6.674238 & -1.058433 & 4.643365 \\
\mathrm{H} & 8.326020 & -1.432659 & 4.148518 \\
\mathrm{H} & 8.650617 & -2.524952 & 2.048838 \\
\mathrm{H} & 5.050262 & -0.219190 & 2.717923 \\
\mathrm{H} & 7.792038 & -3.225359 & -0.190979 \\
\mathrm{H} & 4.187447 & -0.886706 & 0.493294 \\
\mathrm{H} & -1.741163 & 1.939178 & -0.495238 \\
\mathrm{H} & 6.885793 & 1.260533 & 3.660755 \\
\mathrm{H} & -0.068708 & 3.132503 & -1.676701 \\
\mathrm{H} & 2.054409 & 3.219464 & -2.377397
\end{array}
$$



H $\quad 2.863956 \quad 2.292528 \quad-4.584560$
H $\quad 1.278170 \quad 1.573447 \quad-4.856664$
H $\quad 4.507758 \quad 0.098698 \quad-3.847550$
H $\quad 1.795032 \quad-1.612682 \quad-1.072151$
H $\quad 4.199189 \quad-1.690560 \quad-2.003159$

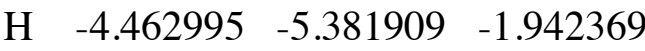
H $\quad-5.025313 \quad-2.574062 \quad-0.780763$
H $\quad-3.898056 \quad-2.920288 \quad-2.080956$
H $\quad-3.216633 \quad-5.720623 \quad 0.401589$
H $\quad-0.704390 \quad-2.518526 \quad 1.576300$
H $\quad-1.217536 \quad-5.004753 \quad 1.836630$
H $\quad-5.785299 \quad-1.064647 \quad 4.266815$
H $\quad-7.788649 \quad 0.298321 \quad 2.357946$
H $\quad-6.771551 \quad 1.137375 \quad 3.523441$
H $\quad-6.030726 \quad 2.357258 \quad 1.137922$
H $\quad-4.541087 \quad-1.522034 \quad 1.792607$
H $\quad-4.033542 \quad 2.033473 \quad-0.402260$
H $\quad-0.692413 \quad 2.619419 \quad-3.167803$
$\begin{array}{llll}\text { O } & -1.812689 & 1.151083 & -1.210069\end{array}$
$\begin{array}{llll}\text { O } & -0.494497 & -0.886314 & -1.008291\end{array}$ 
Table S24. Cartesian coordinates of $\mathrm{OHO}$ in the open-shell singlet state.
Atom $\quad \mathrm{x} \quad \mathrm{y} \quad \mathrm{z}$
$\begin{array}{llll}\mathrm{N} & 0.099048 & 2.501839 & -2.532851\end{array}$
C $\quad 1.359464 \quad 2.942845 \quad-3.176867$
H $\quad 1.162536 \quad 3.846266 \quad-3.767697$
C $\quad 1.992548 \quad 1.868332-4.074947$
C $\quad 2.417427 \quad 0.670150 \quad-3.281589$
N $\quad 1.573558 \quad 0.075196 \quad-2.358322$
C $\quad 3.600700 \quad-0.036297 \quad-3.277168$
C $\quad 2.220870 \quad-0.958170 \quad-1.818753$
N $\quad 3.451094 \quad-1.055780 \quad-2.353960$
H $\quad 1.687917 \quad 4.410451 \quad 1.791468$
C $\quad 1.329545 \quad 5.239097 \quad 2.413215$
H $\quad 1.087218 \quad 4.842018 \quad 3.407567$
$\begin{array}{llll}\text { C } & 0.104280 & 5.909027 & 1.769735\end{array}$
$\begin{array}{llll}\text { C } & -1.094202 & 4.965374 & 1.587229\end{array}$
$\begin{array}{llll}\text { C } & -0.960298 & 3.917866 & 0.476581\end{array}$
$\begin{array}{llll}\text { O } & -1.813581 & 2.961360 & 0.501660\end{array}$
O $\quad-0.064945 \quad 4.057198 \quad-0.405166$
H $\quad-5.903745 \quad-4.398855 \quad-2.235445$
C $\quad-5.118076-4.619361-1.506106$
Н $\quad-5.598632 \quad-5.044729 \quad-0.617284$
$\begin{array}{llll}\text { C } & -4.337994 & -3.339042 & -1.165527\end{array}$
$\begin{array}{llll}\text { C } & -3.233198 & -3.549347 & -0.166476\end{array}$
$\begin{array}{llll}\mathrm{N} & -2.391671 & -2.521546 & 0.250855\end{array}$
$\begin{array}{llll}\text { C } & -2.835215 & -4.712843 & 0.458529\end{array}$
$\begin{array}{llll}\text { C } & -1.510126 & -3.051290 & 1.090758\end{array}$
N $\quad-1.747531 \quad-4.375252 \quad 1.248104$
H $\quad-6.813506-1.916880 \quad 3.093958$
$\begin{array}{llll}\text { C } & -6.731129 & -1.018597 & 3.716231\end{array}$
H $\quad-7.548210 \quad-1.041439 \quad 4.443862$
$\begin{array}{llll}\text { C } & -6.814917 & 0.257395 & 2.866319\end{array}$ 


$$
\begin{aligned}
& \begin{array}{llll}
C & -5.716501 & 0.355553 & 1.846874
\end{array} \\
& \begin{array}{llll}
\mathrm{N} & -5.502523 & 1.495522 & 1.080704
\end{array} \\
& \begin{array}{llll}
\text { C } & -4.736748 & -0.521753 & 1.437934
\end{array} \\
& \begin{array}{llll}
\text { C } & -4.437289 & 1.290943 & 0.269399
\end{array} \\
& \begin{array}{llll}
\mathrm{N} & -3.949816 & 0.071030 & 0.459657
\end{array} \\
& \mathrm{H} \quad 8.545917 \quad 0.891872 \quad 3.160234 \\
& \begin{array}{llll}
\text { C } & 7.767168 & 0.652429 & 3.892001
\end{array} \\
& \mathrm{H} \quad 8.128245 \quad 0.944794 \quad 4.883649 \\
& \begin{array}{llll}
\text { C } & 7.433871 & -0.858987 & 3.875382
\end{array} \\
& \begin{array}{llll}
\text { C } & 6.916405 & -1.317758 & 2.536611
\end{array} \\
& \begin{array}{llll}
\text { C } & 5.643028 & -0.868346 & 2.075585
\end{array} \\
& \begin{array}{llll}
\text { C } & 7.680300 & -2.176394 & 1.699368
\end{array} \\
& \begin{array}{llll}
\text { C } & 5.155788 & -1.237421 & 0.841621
\end{array} \\
& \begin{array}{llll}
\text { C } & 7.215785 & -2.570510 & 0.458338
\end{array} \\
& \begin{array}{llll}
\text { C } & 5.928134 & -2.107848 & -0.034061
\end{array} \\
& \begin{array}{llll}
\text { O } & 5.508442 & -2.453199 & -1.181869
\end{array} \\
& \mathrm{Cu} \quad-0.135432 \quad 0.689336-1.781715 \\
& \mathrm{Cu} \quad-2.236224 \quad-0.581581 \quad-0.351032 \\
& \begin{array}{llll}
\mathrm{H} & -0.211874 & 6.755338 & 2.394587
\end{array} \\
& \begin{array}{llll}
\mathrm{H} & 0.383334 & 6.317753 & 0.792123
\end{array} \\
& \begin{array}{llll}
\mathrm{H} & -1.331036 & 4.434122 & 2.517468
\end{array} \\
& \begin{array}{llll}
\mathrm{H} & -1.993644 & 5.546472 & 1.336759
\end{array} \\
& \mathrm{H} \quad 2.152540 \quad 5.954022 \quad 2.530266 \\
& \begin{array}{llll}
\mathrm{H} & 6.673970 & -1.058574 & 4.643368
\end{array} \\
& \begin{array}{llll}
\mathrm{H} & 8.325709 & -1.433042 & 4.148581
\end{array} \\
& \begin{array}{llll}
\mathrm{H} & 8.649970 & -2.525844 & 2.049173
\end{array} \\
& \begin{array}{llll}
\mathrm{H} & 5.050422 & -0.218633 & 2.717615
\end{array} \\
& \begin{array}{llll}
\mathrm{H} & 7.791332 & -3.226235 & -0.190622
\end{array} \\
& \begin{array}{llll}
\mathrm{H} & 4.187580 & -0.886092 & 0.492982
\end{array} \\
& \begin{array}{lllll}
\mathrm{H} & -1.741049 & 1.939348 & -0.494948
\end{array} \\
& \begin{array}{llll}
\mathrm{H} & 6.885919 & 1.260384 & 3.660809
\end{array} \\
& \text { H } \quad-0.068676 \quad 3.132621 \quad-1.676518 \\
& \text { H } 2.054356 \quad 3.219558 \quad-2.377646
\end{aligned}
$$



$\begin{array}{llll}\mathrm{H} & 2.863602 & 2.292570 & -4.584870\end{array}$
H $\quad 1.277784 \quad 1.573455 \quad-4.856748$
H $\quad 4.507510 \quad 0.098775 \quad-3.847994$
H $\quad 1.795246-1.612372 \quad-1.072002$
H $\quad 4.199230-1.690332 \quad-2.003329$
H $\quad-4.463004-5.381932 \quad-1.942556$
H $\quad-5.025328 \quad-2.574122 \quad-0.780862$
H $\quad-3.898156 \quad-2.920319-2.081135$
H $\quad-3.216526 \quad-5.720646 \quad 0.401322$
$\begin{array}{llll}\mathrm{H} & -0.704089 & -2.518589 & 1.575749\end{array}$
H $\quad-1.217111 \quad-5.0048521 .835982$
H $\quad-5.785305 \quad-1.064973 \quad 4.266992$
$\begin{array}{llll}\mathrm{H} & -7.788613 & 0.297912 & 2.358020\end{array}$
H $\quad-6.771644 \quad 1.137003 \quad 3.523598$
$\begin{array}{llll}\mathrm{H} & -6.030637 & 2.357140 & 1.138463\end{array}$
$\begin{array}{llll}\mathrm{H} & -4.540985 & -1.522247 & 1.792565\end{array}$
H $\quad-4.033466 \quad 2.033582-0.401792$
$\begin{array}{llll}\mathrm{H} & -0.692599 & 2.619647 & -3.167638\end{array}$
$\begin{array}{llll}\text { O } & -1.812603 & 1.151292 & -1.209841\end{array}$
$\begin{array}{llll}\mathrm{O} & -0.494481 & -0.886230 & -1.008410\end{array}$ 